\title{
Cancer immunoediting by melanocyte differentiation antigen-specific T cells determines response to immune checkpoint inhibition
}

Lukas Flatz ( $\square$ lukas.flatz@med.uni-tuebingen.de)

Eberhard-Karls-University Tuebingen, Germany

\section{Sandra Ring}

Kantonsspital St.Gallen

\section{Oltin Pop}

Institute of Immunobiology

Joanna Poźniak

Laboratory for Molecular Cancer Biology

\section{Fiamma Berner}

Institute of Immunobiology

Omar Ali

Institute of Immunobiology

Marie-Therese Abdou

Institute of Immunobiology

\section{Stefan Diem}

Department of Oncology and Hematology

David Bomze

The Hebrew University of Jerusalem

Rebekka Niederer

Institute of Immunobiology

Mirjam Fässler

Institute of Immunobiology

Ewout Landeloos

Laboratory for Molecular Cancer Biology

Florian Rambow

Laboratory for Molecular Cancer Biology

Greet Bervoets

Laboratory for Molecular Cancer Biology

\section{Sandra Freiberger}

Department of Pathology and Molecular Pathology

Thomas Mayr 
Institute of Pathology

\section{Michael Muders}

University of Bonn

\section{Maries van den Broek}

University of Zurich https://orcid.org/0000-0002-9489-3692

\section{Tobias Bald}

QIMR Berghofer https://orcid.org/0000-0003-0061-235X

\section{Jennifer Lansdberg}

Department of Dermatology and Allergology

\section{Dimo Dietrich}

Department of Otolaryngology, Head and Neck Surgery

\section{Joanna Mangana}

Department of Dermatology

\section{Antonio Cozzio}

Department of Dermatology and Allergology

\section{Claus Garbe}

University Medical Center, Eberhard Karls University Tuebingen

\section{Reinhard Dummer}

University Hospital Zurich

\section{Mitchell Levesque}

Department of Dermatology

\section{Wolfram Jochum}

Institute of of Pathology

\section{Burkhard Ludewig}

Kantonsspital St. Gallen https://orcid.org/0000-0002-7685-573X

\section{Oliver Bechter}

Department of General Medical Oncology

\section{Jean-Christophe Marine}

Laboratory for Molecular Cancer Biology

\section{Thomas Tüting}

Laboratory for Experimental Oncology https://orcid.org/0000-0001-7146-0934

Michael Hölzel

Institute of Experimental Oncology

\section{Article}

Keywords: c,ancer immunoediting, cancer immune surveillance, tumor immunogenicity

Posted Date: May 3rd, 2021 
DOl: https://doi.org/10.21203/rs.3.rs-403313/v1

License: (c) (1) This work is licensed under a Creative Commons Attribution 4.0 International License. Read Full License 
Sandra S. Ring ${ }^{1 *}$, Oltin T. Pop ${ }^{1 *}$, Joanna Poźniak ${ }^{2}$, Fiamma Berner ${ }^{1}$, Omar Hasan Ali ${ }^{1,3}$, MarieTherese Abdou ${ }^{1}$, Stefan Diem ${ }^{4,5}$, David Bomze ${ }^{1}$, Rebekka Niederer ${ }^{1,3}$, Mirjam Fässler ${ }^{1}$, Ewout Landeloos $^{2}$, Florian Rambow ${ }^{2}$, Greet Bervoets ${ }^{2}$, Sandra N. Freiberger ${ }^{6}$, Thomas Mayr ${ }^{7}$, Michael Muders $^{7}$, Maries van den Broek ${ }^{8}$, Tobias Bald ${ }^{9}$, Jennifer Landsberg ${ }^{10}$, Dimo Dietrich ${ }^{11}$, Joanna Mangana $^{12}$, Antonio Cozzio ${ }^{3}$, Claus Garbe ${ }^{13}$, Reinhard Dummer ${ }^{12}$, Mitchell P. Levesque ${ }^{12}$, Wolfram Jochum ${ }^{14}$, Burkhard Ludewig ${ }^{1}$, Oliver Bechter ${ }^{15}$, Jean-Christophe Marine ${ }^{2}$, Thomas Tüting ${ }^{16}$, Michael Hölzel ${ }^{17}$, Lukas Flatz ${ }^{1,3,4,12,13}$

* S.S.R. and O.T.P. contributed equally to this work.

Affiliations

${ }^{1}$ Institute of Immunobiology, Kantonsspital St. Gallen, St. Gallen, Switzerland; ${ }^{2}$ Laboratory for Molecular Cancer Biology, VIB Center for Cancer Biology, KU Leuven, Leuven, Belgium; ${ }^{3}$ Department of Dermatology and Allergology, Kantonsspital St. Gallen, Switzerland; ${ }^{4}$ Department of Oncology and Haematology, Kantonsspital St. Gallen, Switzerland; ${ }^{5}$ Department of Oncology and Haematology, Spital Grabs, Switzerland; ${ }^{6}$ Department of Pathology and Molecular Pathology, University and University Hospital Zurich, Switzerland; ${ }^{7}$ Rudolf-Becker-Laboratory for Prostate Cancer Research, Institute of Pathology, University of Bonn Medical Center, Germany; ${ }^{8}$ Institute of Experimental Immunology, University of Zurich, Zurich, Switzerland; ${ }^{9}$ QIMR Berghofer Medical Research Institute, Brisbane, Australia; ${ }^{10}$ Department of Dermatology and Allergy, University of Bonn, Bonn, Germany; ${ }^{11}$ Department of Otolaryngology, Head and Neck Surgery, University Hospital Bonn, Germany; ${ }^{12}$ Department of Dermatology, University Hospital of Zurich, University of Zurich; ${ }^{13}$ Department of Dermatology, University Hospital of Tübingen, Germany; ${ }^{14}$ Institute of Pathology, Kantonsspital St. Gallen, Switzerland; ${ }^{15}$ Department of General Medical Oncology UZ Leuven, Belgium; ${ }^{16}$ Laboratory for Experimental Dermatology, Department of Dermatology, University Hospital Magdeburg, Magdeburg, Germany;

${ }^{17}$ Institute of Experimental Oncology, University Hospital Bonn, University of Bonn, Germany.

Corresponding author: Lukas Flatz, email: lukas.flatz@med.uni-tuebingen.de

Short title: Cancer immunoediting is associated with checkpoint inhibitor response 


\section{Abstract}

35 T cells are critical in cancer immune surveillance but they can also shape tumor immunogenicity, 36 described as cancer immunoediting ${ }^{1,2}$. Melanoma patients commonly harbor $\mathrm{T}$ cells recognizing 37 melanocyte differentiation antigens (MDAs) $)^{3-6}$. However, the roles of MDA-specific T cells in 38 shaping melanoma immunogenicity and the response to immune checkpoint inhibition remain 39 elusive. Here, we prospectively profiled peripheral $\mathrm{CD}^{+} \mathrm{T}$ cells from 27 stage IV patients before initiation of checkpoint inhibitor therapy. Clinical failure was associated with increased MDAspecific $\mathrm{CD}^{+} \mathrm{T}$ cells and reduced tumor MDA expression pretreatment. In nonresponders, decreased tumor MDA expression was concomitant with a dedifferentiated melanoma phenotype. We confirmed in 30 stage III patients that individuals with relapse disease during adjuvant antiPD-1 therapy demonstrated a significantly higher incidence of dedifferentiated tumors pretreatment than individuals without recurrence. Thus, MDA-directed $\mathrm{CD}^{+} \mathrm{T}$ cells are associated with a dedifferentiated phenotype and reduced clinical response to checkpoint inhibitor therapy suggesting immunoediting as an important resistance mechanism. 
Cancer immunotherapies targeting the inhibitory checkpoint molecules PD-1 and CTLA-4 harness the patient's antitumor immunity and the presence of $\mathrm{CD}^{+} \mathrm{T}$ cell infiltrates in tumors is generally considered a positive correlative factor for response to checkpoint inhibitor therapy ${ }^{7,8}$. While tumor-specific $\mathrm{CD}^{+} \mathrm{T}$ cells play a critical role in cancer immune surveillance ${ }^{9}$, they can also shape tumor immunogenicity to evade immune-mediated killing, a process known as cancer immunoediting ${ }^{1,2}$. One mechanism of cancer immunoediting was previously shown in mouse models in which adoptive transfer of $\mathrm{CD}^{+} \mathrm{T}$ cells specific for melanosomal antigens elicited melanoma dedifferentiation in response to $\mathrm{T}$ cell-derived inflammatory cytokines and resulted in the loss of $\mathrm{T}$ cell-mediated tumor control ${ }^{10-12}$. However, evidence of inflammation-induced dedifferentiation ${ }^{13}$ in humans is scarce and hitherto derived from in vitro human cell lines experiments $^{14,15}$, transcriptomic studies ${ }^{16,17}$ or case reports ${ }^{18}$. In one patient, adoptive transfer of MART-1-specific $\mathrm{CD}^{+} \mathrm{T}$ cells induced inflammation in the tumor microenvironment resulted in gradual loss of expression of MART-1 and other melanocytic antigens in relapsing tumors ${ }^{18}$. Although melanocyte differentiation antigen (MDA)-specific T cells have been studied for a long period in melanoma patients ${ }^{4,19,20}$, surprisingly little is known about the phenotypic co-evolution between MDA-specific $\mathrm{T}$ cells with melanoma respectively their influence in the context of immune checkpoint inhibitor treatment.

A high proportion of melanoma patients commonly harbor $\mathrm{CD}^{+} \mathrm{T}$ cells recognizing tumorassociated antigens such as MDAs ${ }^{5,6}$, cancer-testis antigens $(\mathrm{CTA})^{4,21}$ or antigens derived from tumor-specific mutations (neo-antigens) $)^{22}$ but their role in immune checkpoint therapy has not been resolved. Vitiligo is elicited by MDA-specific $\mathrm{CD}^{+} \mathrm{T}$ cells and represents a positive correlative factor for clinical responses to immunotherapies in melanoma ${ }^{23-25}$. To assess MDAand CTA-specific $\mathrm{CD}^{+} \mathrm{T}$ cell responses in the context of checkpoint inhibitor therapy, we prospectively enrolled 27 patients with stage IV melanoma treated with an anti-PD-1 antibody 
either alone or in combination with an anti-CTLA-4 antibody (St. Gallen (SG) cohort, Fig. 1a and Extended Data Table 1). All patients were treatment naïve for anti-PD-1 therapy. Consistent with published trials, $44 \%$ of the patients in our cohort were responders, while $56 \%$ of the patients in our cohort did not show an objective clinical response at 12 weeks according to Response Evaluation Criteria in Solid Tumors (RECIST) $1.1^{26-29}$ (Extended Data Table 1, Extended Data Fig. 1a). To examine $\mathrm{CD}^{+} \mathrm{T}$ cell responses, we stimulated peripheral blood mononuclear cells (PBMCs) collected before initiation of checkpoint inhibitor therapy with separate overlapping peptide pools representing the full-length sequence of the MDAs tyrosinase (TYR), MART-1, gp100 and tyrosinase-related protein 2 (TRP2) and the CTAs MAGE-A1, MAGE-A3, MAGE-A4 and NY-ESO-1 (Fig. $1 \mathrm{~b}$ and Extended Data Fig. 1b). We found that the activity of antigenexperienced CD45RA ${ }^{\text {low }} \mathrm{CD}^{+} \mathrm{T}$ cells was commonly directed against the MDAs TYR (21 of 27 patients), MART-1 (7 of 27 patients), and gp100 (6 of 27 patients), as indicated by the expression patterns of the cytokines IFN- $\gamma$ and TNF- $\alpha$ (Fig. 1c, Extended Data Fig. 1c and Extended Data Fig. 2a). Fewer T cell responses were observed against the MDA TRP2 (2 of 27 patients) and the CTAs MAGE-A1 (1 of 27 patients), MAGE-A3 (1 of 27 patients), MAGE-A4 (4 of 27 patients), and NY-ESO-1 (4 of 27 patients) (Extended Data Fig. 1c to e). Therefore, within this study we further focused on $\mathrm{CD}^{+} \mathrm{T}$ cells specific for the MDAs TYR, MART-1, and gp100. Interestingly, substantially higher proportions of nonresponders (NRs) than responders (Rs) showed increased IFN $-\gamma^{+}$and $\mathrm{TNF}-\alpha^{+} \mathrm{CD}^{+} \mathrm{T}$ cell responses against TYR, MART-1 and gp100 (Fig. 1c and d, Extended Data Fig. 1c and d and Extended Data Fig. 2a and b). Moreover, the nonresponders were characterized by a broader MDA-specific $\mathrm{CD}^{+} \mathrm{T}$ cell response, defined as cytokine-expressing $\mathrm{CD}^{+} \mathrm{T}$ cells against any of the melanocytic antigens TYR, MART-1 or gp100 (Fig. 1c and e and Extended Data Figure 2a and c). Likewise, the frequency of cytokine-producing $\mathrm{CD}^{+} \mathrm{T}$ cells specific for the three MDAs combined was significantly higher in the nonresponders compared to responders (Fig. 1c and $\mathrm{f}$ and Extended Data Fig. 2a and d). In sum, our data show that 
nonresponders exhibit a higher proportion of MDA-specific $\mathrm{CD}^{+} \mathrm{T}$ cells prior to checkpoint inhibitor therapy.

A $\mathrm{T}$ cell-mediated immunoselection process can result in the outgrowth of tumor cells lacking expression of immunodominant antigens ${ }^{30}$. To investigate the relationship between MDA-specific

$102 \mathrm{~T}$ cells and MDA tissue expression, we performed quantitative immunohistochemical (IHC)

103 analysis for TYR, MART-1 and gp100 of pretreatment tumor samples from 19 patients in the SG 104 cohort $(\mathrm{R}=10, \mathrm{NR}=9$; Fig. $2 \mathrm{a}$ and $\mathrm{b})$. The percentage of tumor cells expressing each of these 105 antigens was assessed as outlined in Extended Data Figure 3. Tumor cells of responders showed a 106 higher frequency of expression of the individual MDAs TYR, MART-1 and gp100 compared to 107 nonresponders (Fig. 2c and d). Moreover, expression levels of individual MDAs were not only 108 decreased in nonresponders but we observed a broad loss of all melanocytic proteins in 109 nonresponders (7 of 9 nonresponder, patients 1, 8, 14, 18, 19, 20 and 21) compared to responders 110 (9 of 10 responders, patients $2,4,5,11,12,15,17,22,25$ ) as described by the reduced breath of 111 the expression of TYR, MART-1 and gp100 combined (Fig. 2c). Finally, Spearman's correlation 112 analysis revealed an inverse correlation between tumor MDA expression in pretreatment biopsies 113 and MDA-specific $\mathrm{CD}^{+} \mathrm{T}$ cell responses in blood (Fig. $2 \mathrm{c}$ and e, Spearman $\mathrm{r}=-0.5666, \mathrm{P}=0.01$ ).

114 These observations showed that the broad loss of melanocytic antigen expression together with 115 increased MDA-specific $\mathrm{CD}^{+} \mathrm{T}$ cells in the periphery are associated with an impaired response 116 to checkpoint inhibitor therapy. Thus, MDA-directed $\mathrm{T}$ cell responses may have an 117 underappreciated yet crucial role in shaping melanoma immunogenicity and affecting the clinical 118 outcome to immune checkpoint inhibitors.

119 A critical role of $\mathrm{T}$ cell responses against MDAs in melanoma emanates from therapies using 120 adoptive transfer of either ex vivo expanded tumor-infiltrating lymphocytes or TCR-engineered $121 \mathrm{~T}$ cells recognizing MDAs ${ }^{31,32}$. In mouse melanoma models, adoptive transfer of $\mathrm{T}$ cells targeting 122 a melanosomal antigen compared to transfer of $\mathrm{T}$ cells against an oncogenic antigen, provoked 
123 profound dedifferentiation ${ }^{11}$. Therefore, dedifferentiation represents an important evasion 124 mechanism in response to $\mathrm{T}$ cell-mediated immune pressure ${ }^{12,13}$. In our study, we have observed 125 high pre-existing MDA-specific $\mathrm{CD}^{+} \mathrm{T}$ cells concomitant with a broad loss of melanocytic 126 antigen expression predominantly in nonresponders. Next, we investigated whether tumors with 127 reduced MDA tissue antigen levels also exhibited a dedifferentiated phenotype rather than only 128 selective downregulation of melanocytic antigens. Melanoma dedifferentiation proceeds along a 129 differentiation trajectory that can be subclassified into four subtypes ranging from melanocytic 130 and transitory to neural crest-like and undifferentiated ${ }^{16,33}$. To assess whether progression along 131 the dedifferentiation trajectory described by Tsoi et al. in melanoma cell lines ${ }^{33}$ can be discerned 132 in patient samples, we performed single-cell RNA sequencing (scRNA-seq) of melanoma 133 metastases collected from 15 stage III or stage IV patients (Cohort Leuven; Extended Data Table $1342 ; 14$ of the 15 patients were drug-naive). Unsupervised clustering of all 15 tumor lesions 135 combined using Harmony integration and Uniform Manifold Approximation and Projection 136 (UMAP) revealed 11 distinct clusters (Fig. 3a and Extended Data Fig. 4a and b). We leveraged the 137 gene signatures for the melanoma differentiation subtypes identified by recent transcriptomic 138 analysis $^{33,34}$ and created corresponding scores for each subtype (Extended Data Tables 4 and 5).

139 Based on these scores, we detected different melanoma differentiation cell states in this patient 140 cohort. Tumor cells in clusters $1,4,5,6,8$ and 10 showed a melanocytic (i.e., differentiated) 141 phenotype with a high pigmentation signature, while tumor cells in clusters 0,2 and 3 and 7 were 142 in a transitory state with reduced pigmentation levels (Fig. 3b and Extended Data Fig. 4c and d).

143 In contrast, melanoma cells in clusters 2, 3, 7 and 9 were identified as dedifferentiated phenotype 144 as characterized by increased expression of genes associated with a neural crest-like cell signature 145 (cluster 2, 7 and 9) or a an undifferentiated (i.e., "invasive") phenotype (cluster 3 and 9) (Fig. 3b). 146 Likewise, cells in clusters 2, 3, 7 and 9 demonstrated a reduced pigmentation signature as well as 147 MDA expression and increased expression levels of neural crest stem cell (NCSC) and epithelial- 
mesenchymal transition (EMT) markers (Extended Data Fig. 4c and d). In addition, levels of the transcription factor $M I T F$, the master regulator of melanocytic differentiation ${ }^{35,36}$, were decreased in melanoma cells in clusters 2, 3 and 9 (Fig. 3c), all of which harbor the dedifferentiated melanoma phenotype (Fig. 3b). Moreover, we observed increased expression of the receptor

152 tyrosine kinase EGFR in clusters 3 and 9, while tumor cells from clusters 2 and 7 expressed the 153 neural crest marker $N G F R$ (Fig. 3c), both marker are linked to a dedifferentiated melanoma 154 phenotype $\mathrm{p}^{10,14,37,38}$. Overall, this single-cell transcriptomic analysis in treatment-naïve clinical samples established the presence of melanoma cells exhibiting distinct differentiation statuses ranging from cells with either a melanocytic differentiated phenotype to a dedifferentiated 157 phenotype with reduced melanocytic antigen expression.

158 Next, we sought to investigate whether tumor cells from patients in the SG cohort not only exhibited reduced expression of melanocytic antigens but show a dedifferentiated phenotype. We performed quantitative IHC analysis of the 19 available biopsies obtained prior to treatment (Fig. 3d-g, Fig 2a, Extended Data Fig 3) and examined tissue expression of MITF, EGFR and NGFR, 162 markers associated with dedifferentiated tumors according to our scRNA-seq data (Fig. 3).

163 Strikingly, our IHC analysis revealed significantly reduced expression of MITF and markedly 164 increased frequency of tumor cells expressing EGFR in nonresponders compared to responders, 165 whereas the expression of NGFR was not different (Fig. 3d and e, Extended Data Fig. 5a to c). We 166 further defined tumor cells with low MITF expression (MITF ${ }^{\text {low }}$ ) and high EGFR expression 167 (EGFR $\left.{ }^{\text {high }}\right)$ as dedifferentiated melanomas based on threshold values determined for each protein 168 by receiver operator characteristic (ROC) curves (Fig. 3e, green boxes). Dedifferentiated MITF ${ }^{\text {low }}$ 169 EGFR $^{\text {high }}$ tumors were identified exclusively in nonresponders (8 out of 9 ), while tumors from 170 responders showed a uniformly differentiated and melanocytic phenotype (Fig. 3e and Fig 2c and 171 and f, Extended Data Figure 5d and e). Moreover, compared to patients with differentiated tumors, 172 patients with dedifferentiated tumors demonstrated significantly diminished progression-free 
173 survival (Fig. 3g). Thus, melanoma patients with reduced MDA expression not only selectively 174 downregulated these antigens but rather exhibited a dedifferentiated phenotype which was 175 exclusively found in nonresponders.

176 Finally, to strengthen our findings that patients with dedifferentiated melanomas are associated 177 with poor outcome to checkpoint inhibitor treatment, we obtained tumor biopsies from 30 stage 178 III melanoma patients before initiation of adjuvant checkpoint inhibitor therapy (Zurich (ZH) 179 cohort, Extended Data Table 3). Adjuvant anti-PD-1 therapy after surgical resection of regional 180 lymph node metastases has become the standard of care in melanoma patients, resulting in 181 significantly longer recurrence-free survival than placebo treatment ${ }^{39,40}$. In this independent 182 cohort, $37 \%$ of patients developed recurrence within the observed time (Relapses; Rel), whereas 183 63\% remained disease-free (Disease-Free; DF) (Fig. 4a, Extended Data Fig. 6a). We performed 184 IHC analysis of the collected biopsies and quantified the expression of MITF, EGFR, NGFR (Fig. $1854 \mathrm{~b}$ and $\mathrm{c}$ and Extended Data Fig. $6 \mathrm{~b}$ to d). In this validation cohort, $47 \%$ of patients (14 out of 30) 186 presented with a MITF ${ }^{\text {low }}$ EGFR $^{\text {high }}$ and/ or MITF ${ }^{\text {low }}$ NGFR $^{\text {high }}$ dedifferentiated tumor phenotype. 187 Strikingly, individuals with relapsed disease were characterized by a significantly higher incidence 188 of dedifferentiated tumors than patients without recurrence (Fig. 4c and d, Extended Data Figure $1896 \mathrm{~b}$ to f). Accordingly, adjuvant anti-PD-1 treated patients with a dedifferentiated tumor phenotype 190 exhibited significantly shorter progression-free survival than those with a differentiated tumor 191 phenotype (Fig. 4e). Together, these data demonstrate that patients with a dedifferentiated 192 melanoma prior to treatment initiation are associated with therapeutic resistance to adjuvant 193 immune checkpoint therapy. Importantly, we have shown that dedifferentiated melanomas impacts 194 therapy response independent of the stage of the tumor.

195 In this study, immune profiling of peripheral T cells revealed an interrelationship among MDA196 directed $\mathrm{CD}^{+} \mathrm{T}$ cell responses and reduced expression of the MDAs TYR, MART-1 and gp100 197 in the tumor tissue. Additionally, MDA downregulation was correlated with an increased incidence 
198 of a dedifferentiated phenotype and poor clinical outcome following immune checkpoint 199 inhibition. Taken together, we showed in two independent cohorts of patients with different tumor stages (stages III and IV) that a differentiated phenotype is negatively associated with the response 201 to immune checkpoint inhibitor blockade.

202 Increased T cell infiltrates into tumors ${ }^{7,8}$ as well as T cell-mediated adverse events, such as vitiligo 203 which is elicited by MDA-specific $\mathrm{CD} 8^{+} \mathrm{T}$ cells ${ }^{23-25}$, represent a positive correlation with clinical outcome. However, these tumor-specific immune responses can, at the same time, promote cancer immunoediting and affect clinical response to treatment. During the course of disease, cancer immunoediting by MDA-specific $\mathrm{CD}^{+} \mathrm{T}$ cells may drive the evolution of tumor cells resulting in 207 reduced MDA expression and, ultimately, the loss of melanocytic identity. Tumors in patients may 208 fall into one of the three phases of the immunoediting process - elimination, equilibrium or escape ${ }^{2}$ - and can result in different clinical outcomes to checkpoint inhibitor treatment.

210 Immune checkpoint blockade with anti-PD-1 re-invigorates pre-existing tumor-specific $\mathrm{CD}^{+} \mathrm{T}$ 211 cells in a large proportion of melanoma patients but only $40 \%$ of the patients respond clinically ${ }^{41}$.

212 Thus, based on our data, we hypothesize that release of $\mathrm{CD}^{+} \mathrm{T}$ cells targeting melanocytic 213 antigens upon PD-1 therapy results in immune-mediated elimination of melanoma cells in patients 214 with differentiated tumor phenotypes. In contrast, releasing the brakes on pre-existing MDA215 specific $\mathrm{CD}^{+} \mathrm{T}$ cells in patients with dedifferentiated tumors may be less effective because (i) 216 these tumors have lost their melanocytic identity and thus the expression of melanocytic antigens 217 during the course of disease and (ii) MDA-specific $\mathrm{CD}^{+} \mathrm{T}$ cells may outcompete the expansion 218 of $\mathrm{T}$ cells with other specificities. Kvistborg et al. have studied $\mathrm{CD}^{+} \mathrm{T}$ cell responses to 219 melanoma-associated epitopes in patients receiving anti-CTLA-4 therapy using HLA-*0201220 restricted tetramers. Within their study, they have identified pre-existing melanoma-specific $\mathrm{T}$ cell 221 prior to treatment but did not relate them to therapeutic outcome to immune checkpoint blockade ${ }^{19}$. 222 In our study, we have employed an HLA-independent methodological approach using overlapping 
223 peptide pools which unveiled a high level of detectable MDA- and to a lesser extent CTA-specific $224 \mathrm{CD}^{+} \mathrm{T}$ cells prior to checkpoint inhibitor treatment and markedly increased frequencies of the 225 MDAs TYR, MART-1 and gp100 were detected in nonresponders compared to responders. 226 Therefore, our study suggests that MDA-directed T cells have a more profound impact on different 227 melanoma therapies than currently assumed. Understanding the co-evolution of melanoma cells in 228 response to immunological pressure by $\mathrm{T}$ cells targeting melanocytic antigens is critical to improve 229 the outcome of patients treated with cancer immunotherapies and in the design of new treatments 230 targeting dedifferentiated tumors.

231 Our study is not without caveats. First, it characterizes $\mathrm{CD}^{+} \mathrm{T}$ cell responses against a limited 232 number of melanoma-associated antigens without considering $\mathrm{CD}^{+}$or $\mathrm{CD} 4^{+} \mathrm{T}$ cell responses 233 specific for other antigens. Second, our study cohorts are relatively small. Still, we have validated 234 and combined our findings in three independent patient cohorts and these findings are based on 235 strong data generated in mouse models ${ }^{10,11,16}$. Third, in almost all patients we only analyzed one 236 available tumor lesion obtained at a time point close to initiation of checkpoint inhibitor therapy 237 and the collection of PBMCs. However, further studies should evaluate the tumor phenotype and 238 the corresponding $\mathrm{T}$ cell responses during the time course of the disease as well as in multiple 239 lesions. Lastly, we suggest that immune-mediated dedifferentiation is an important resistance 240 mechanism for immune checkpoint inhibitor therapy in melanoma; yet this resistance mechanism 241 may be transferable to other tumor entities. 
a

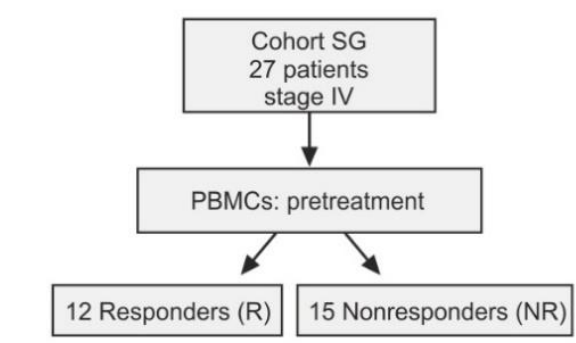

b

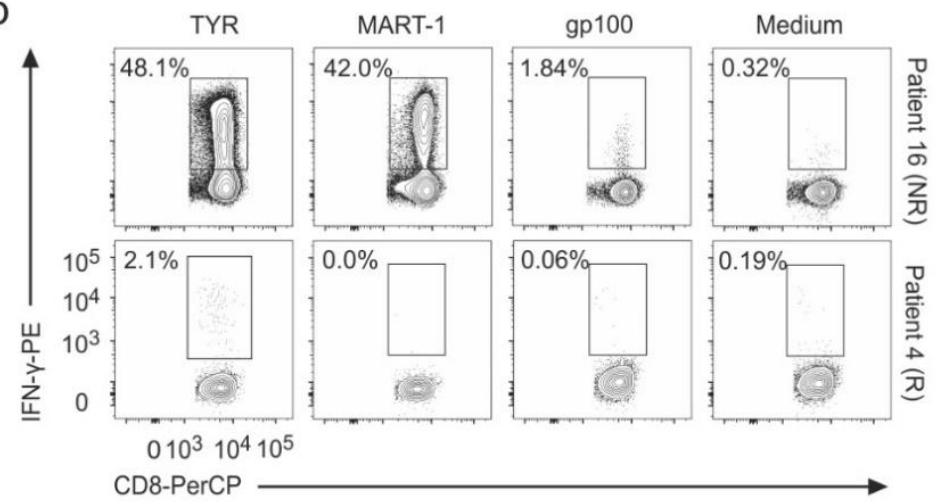

C

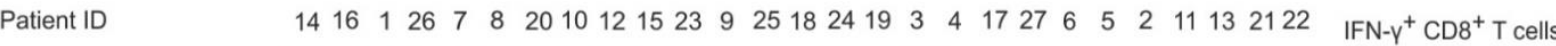
TYR MART-1 gp100

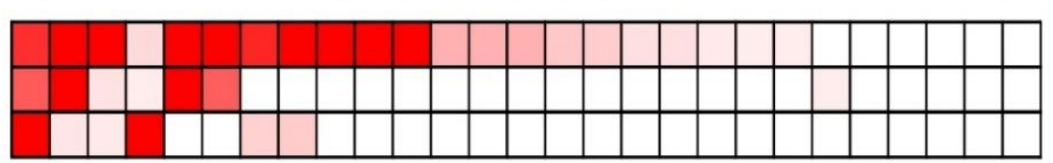
$0 \%>15 \%$

Breadth of $\mathrm{T}$ cell response

Response to $\mathrm{Cl}$ therapy
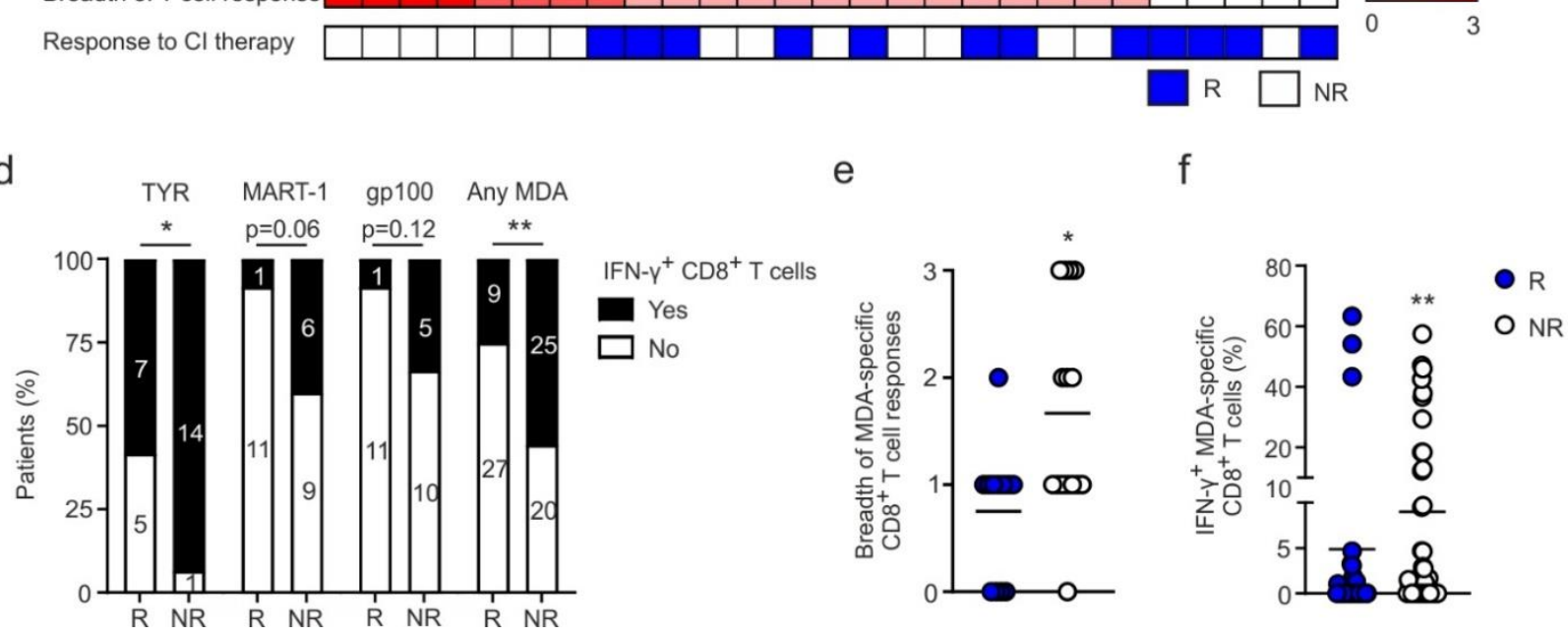

Figure 1. Levels of pre-existing $\mathrm{MDA}$-specific $\mathrm{CD8}^{+} \mathrm{T}$ cells in melanoma patients receiving cells (PBMCs) were obtained from 27 patients with stage IV melanoma prior to checkpoint inhibitor therapy. Clinical responders ( $\mathrm{R} ; \mathrm{N}=12$ ) and nonresponders (NR; $\mathrm{N}=15)$ were determined at 12 weeks. b-f, $\mathrm{CD}^{+} \mathrm{T}$ cell responses against the melanocyte differentiation antigens (MDAs) TYR, MART- 1 and gp100 after in vitro stimulations of PBMCs with overlapping 15-mer peptide pools for each individual antigen for 10 days as measured by IFN- $\gamma$ expression. $\mathbf{b}$, Representative FACS plots of samples from patient 16 (NR) and patient $4(\mathrm{R})$. c, Heatmaps summarizing the frequency of IFN- $\gamma$-producing $\mathrm{CD}^{+} \mathrm{T}$ cells for the indicated antigens. The color scale represents the frequency of IFN $-\gamma^{+} \mathrm{CD} 3^{+} \mathrm{CD} 8^{+} \mathrm{CD} 45 \mathrm{RA}{ }^{\text {low }} \mathrm{T}$ cells. The breadth of the $\mathrm{CD} 8^{+} \mathrm{T}$ cell response was defined as the number of the individual IFN- $\gamma^{+} \mathrm{CD} 8^{+} \mathrm{T}$ cells against the MDAs TYR, MART-1 and gp100, as indicated by the detection of IFN- $\gamma^{+} \mathrm{CD} 8^{+} \mathrm{T}$ cell responses, ranging from 0 (none) to 3 (all). d, Proportions of $\mathrm{R}$ and NR with MDA-specific $\mathrm{CD} 8^{+} \mathrm{T}$ cell responses to the individual MDAs or any MDA. e, Summary of the breadth of MDA-specific CD8 ${ }^{+} \mathrm{T}$ cells in $\mathrm{R}$ and NR as shown for individual patients as shown in panel c. f, Frequencies of IFN- $\gamma^{+} \mathrm{CD} 8^{+} \mathrm{T}$ cells against any MDA (TYR, MART-1 or gp100) in R vs NR. Dots represent individual patients, and lines indicate the mean values (e and f). Statistical analysis was performed using the chi-square test (d) and Mann-Whitney test (e and $\mathrm{f}) ; * \mathrm{P}<0.05, * * \mathrm{P}<0.01, * * * \mathrm{P}<0.001$. 
a

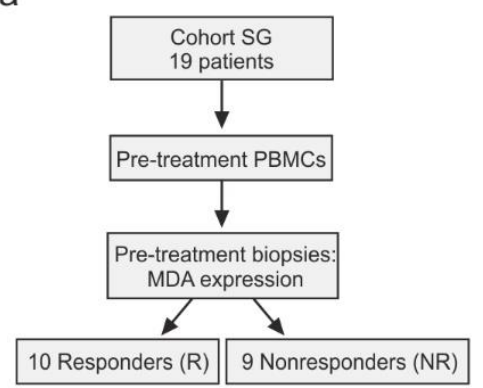

b

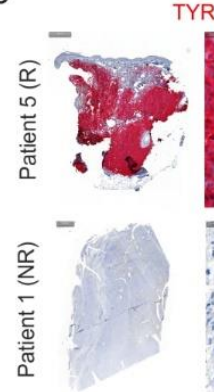

YR/H
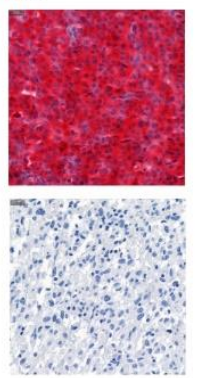
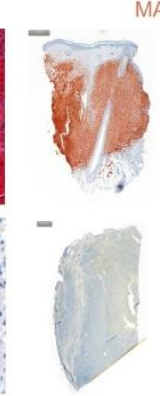

MART-1/H
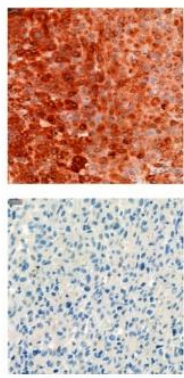

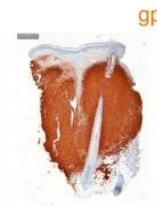

gp 100/H
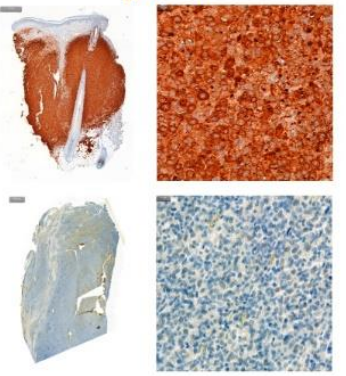

C

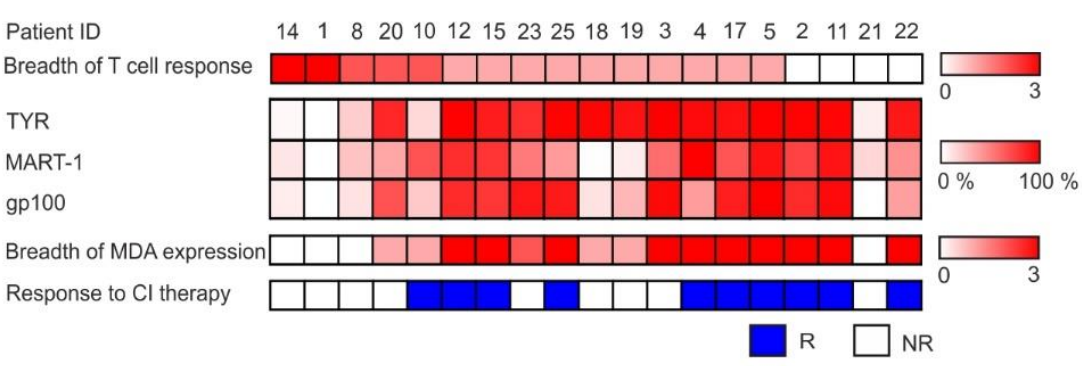

d

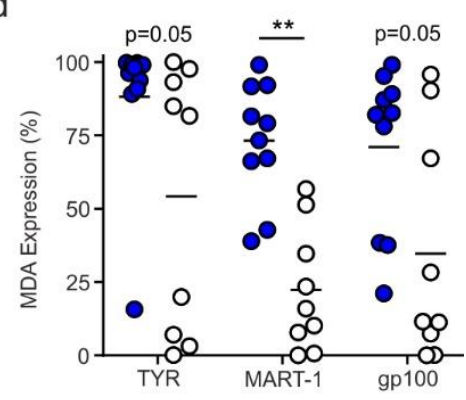

e

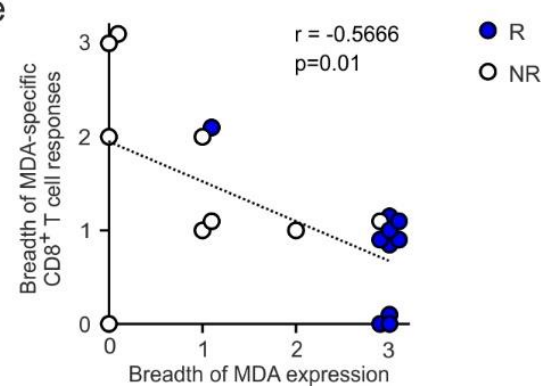

Figure 2. MDA tissue expression inversely correlates with peripheral MDA-specific $\mathrm{CD8}^{+} \mathbf{T}$ cells. a, In 19 of the patient from the SG cohort, tumor biopsies obtained before initiation of checkpoint inhibitor therapy and matched PBMCs from Fig. 1 were analyzed. The expression of the MDAs TYR, MART-1 and gp100 was analyzed using quantitative IHC analysis of biopsy tissue. b, Representative images for the indicated antigens in patient $5(\mathrm{R})$ and patient $1(\mathrm{NR})$. The frequency of tumor cells expressing each of these antigens was assessed as outlined in Extended Data Figure 3. Hematoxylin $(\mathrm{H})$ staining to identify the cell nucleus. Scale bars are 500 and $2000 \mu \mathrm{m}$ for the general images (top and bottom images) and 20 $\mu \mathrm{m}$ for the magnified images. c, Heatmaps summarizing the frequency of melanoma cells stained positive for the MDAs TYR, MART-1 and gp100. The breadth of MDA expression was defined as the number of MDAs expressed per patient sample ranging from 0 (none) to 3 (all) as defined by values from ROC curves for each individual antigen. The breadth of T cell response (data from Fig. 1) is specified as the number of individual MDAs recognized, as indicated by the detection of IFN- $\gamma^{+} \mathrm{CD}^{+} \mathrm{T}$ cell responses, ranging from 0 (none) to 3 (all). d, Frequencies of melanoma cells showing positive staining for each of the MDAs TYR, MART-1 and gp100 in the analyzed tissue slides between R and NR. Dots represent individual patients, and lines indicate mean values. e, Correlation between the breadth of MDA expression and that of MDAspecific $\mathrm{CD} 8^{+} \mathrm{T}$ cell responses. Dots represent individual patients. Statistical analysis was performed using the Mann-Whitney test (d) and Spearman's correlation (e); $* \mathrm{P}<0.05, * * \mathrm{P}<0.01, * * * \mathrm{P}<0.001$. 
a

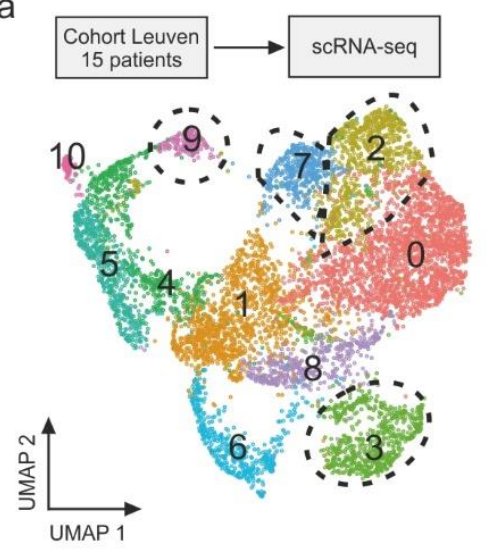

C

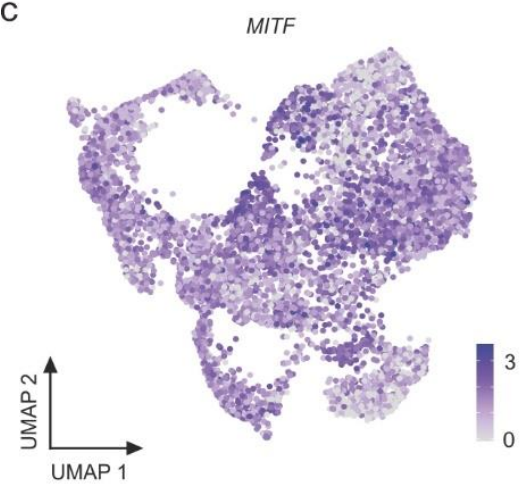

b
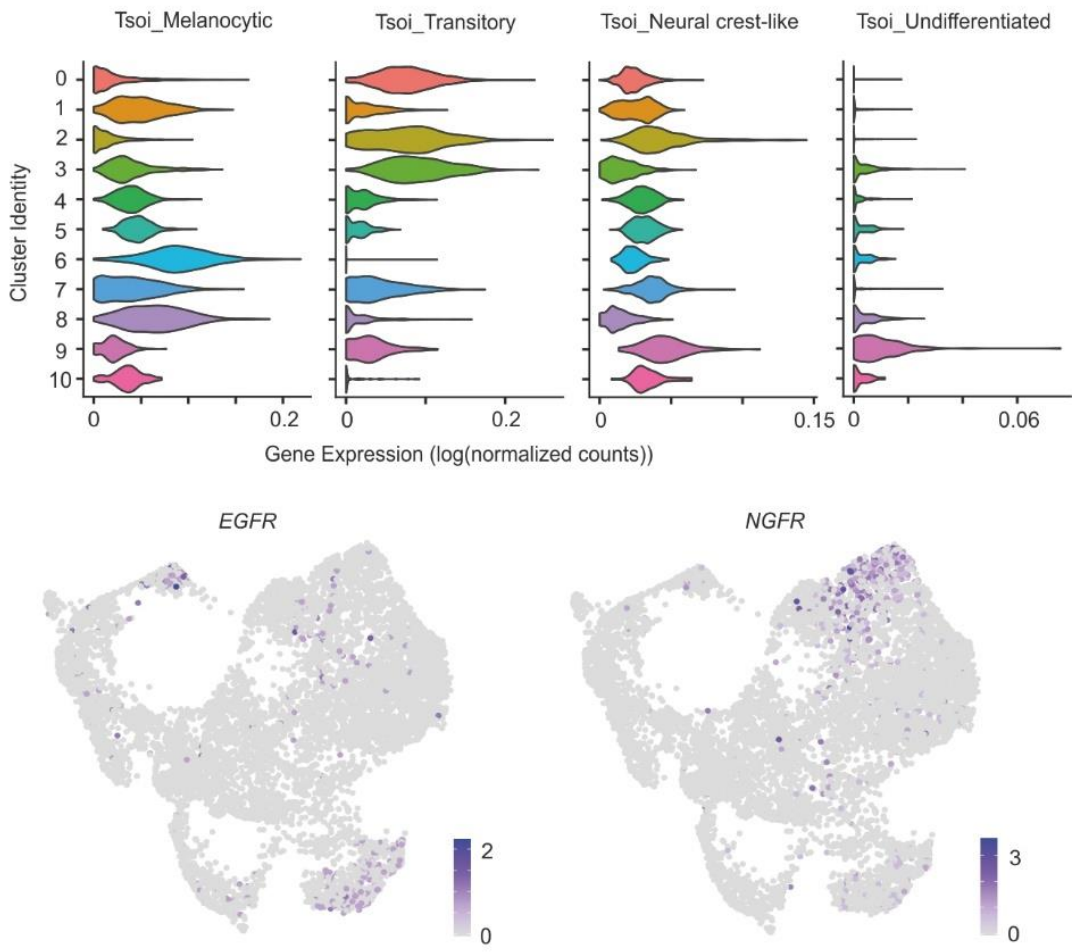

d

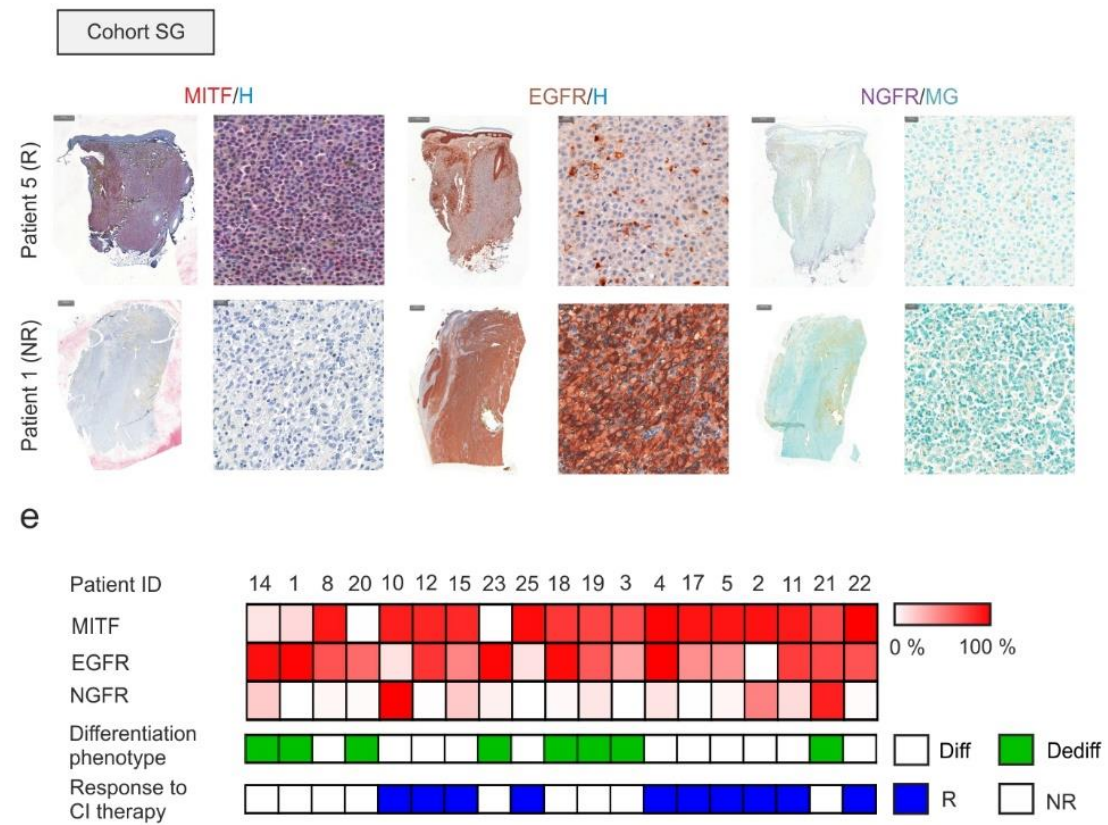

f

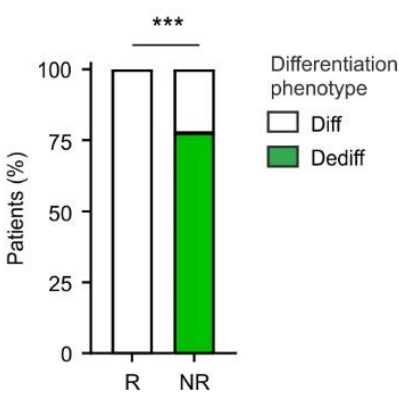

g

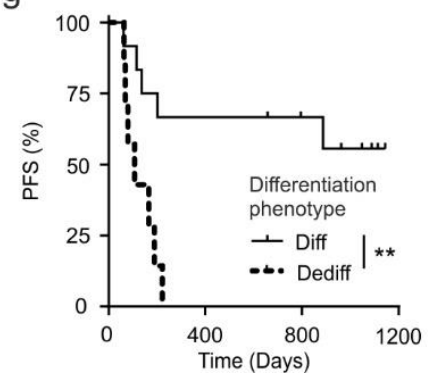

Figure 3. The dedifferentiated melanoma phenotype predicts patient outcome in response to checkpoint inhibitor therapy. a-c, We collected melanoma metastases from 15 stage III or stage IV treatment-naïve patients and performed single-cell RNA-seq analysis of tumor cells (Leuven cohort). a, UMAP plot displaying cluster assignment. Highlighted clusters harbor tumor cells with a dedifferentiated phenotype (cluster 2, 3, 7 and 9) whereas the other cluster exhibit a melanocytic and differentiated phenotype. b, Violin plots based on the Tsoi et el. (2018) gene signatures for melanocytic, transitory, neural crest-like and undifferentiated melanoma phenotypes. c, UMAP plots showing the gene expression of 
MITF, EGFR and NGFR. d-g, In the SG cohort, the expression of MITF, EGFR and NGFR was assessed in tumor biopsies obtained before initiation of checkpoint inhibitor therapy by quantitative IHC analysis. d, Representative images for the indicated antigens in patient $5(\mathrm{R})$ and patient 1 (NR). The frequency of tumor cells expressing each of these antigens was assessed as outlined in Extended Data Figure 3. Hematoxylin (H) or Methyl Green (MG) staining to identify the cell nucleus. Scale bars are 500 and 2000 $\mu \mathrm{m}$ for the general images (top and bottom images) and $20 \mu \mathrm{m}$ for the magnified images. e, Heatmaps summarizing the frequencies of melanoma cells showing positive staining for MITF, EGFR or NGFR using quantitative IHC analysis. The dedifferentiated melanoma phenotype (Dediff) was defined as MITF low EGFR $^{\text {high }}$ or MITF ${ }^{\text {low }}$ NGFR $^{\text {high }}$ as based on threshold values from ROC curves for each individual protein. f, Proportions of patients in the 19 patients from the SG cohort with a dedifferentiated phenotype in the R and NR groups. g, Progression-free survival of patients with a dedifferentiated or differentiated melanoma phenotype. Statistical analysis was performed using the chi-square test (f) or the Mantel-Cox log-rank test $(\mathrm{g}) ; * \mathrm{P}<0.05, * * \mathrm{P}<0.01, * * * \mathrm{P}<0.001$ 
a

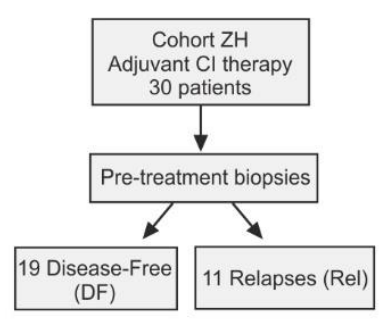

b
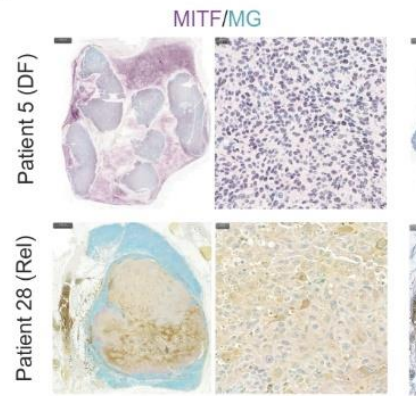
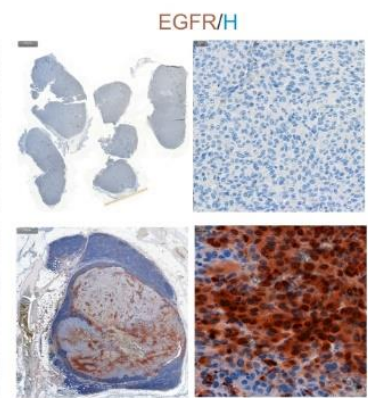

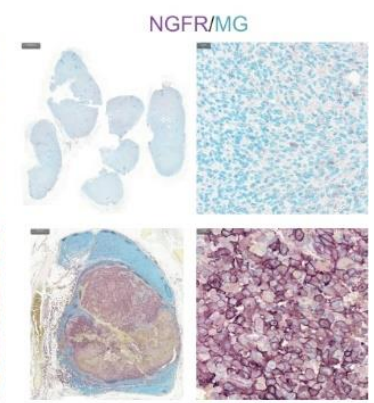

C

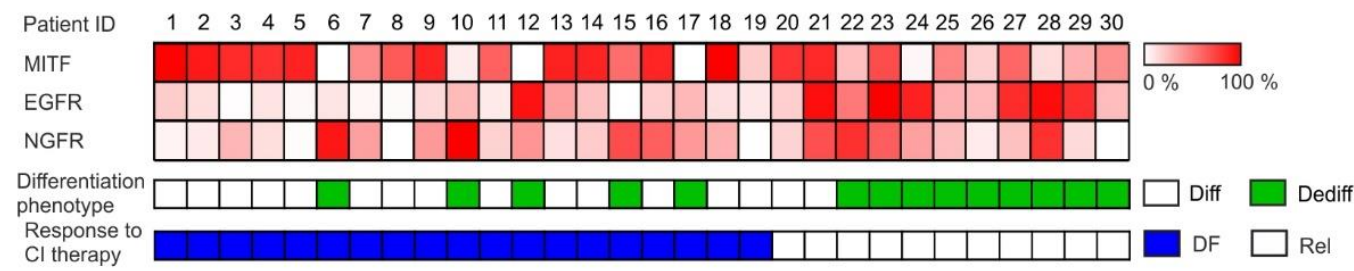

d

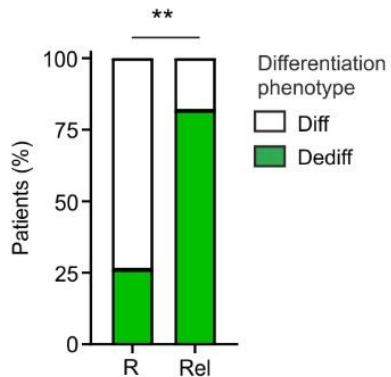

e

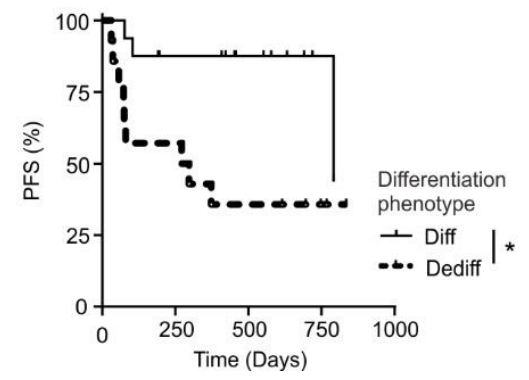

Figure 4. The dedifferentiated melanoma phenotype predicts patient outcome in response to adjuvant anti-PD-1 therapy. a, In the Zurich (ZH) adjuvant cohort, tumor biopsies were obtained before initiation of adjuvant anti-PD-1 treatment. Disease-Free patients (DF; R; $n=19$ ) and patients who experienced relapse (Rel; $\mathrm{n}=11$ ) were determined at the first PET-CT scan within 3 months after start of therapy. b, Representative images showing the expression of MITF, EGFR and NGFR in patient 5 (DF) and patient 28 (Rel). The frequency of tumor cells expressing each of these antigens was assessed as outlined in Extended Data Figure 3. Hematoxylin (H) or Methyl Green (MG) staining to identify the cell nucleus. Scale bars are 1000 and $2000 \mu \mathrm{m}$ for the general images (top and bottom images) and $20 \mu \mathrm{m}$ for the magnified images. c, Heatmaps summarizing the frequency of tumor cells stained positive for MITF, EGFR and NGFR using quantitative IHC analysis. The dedifferentiated phenotype (Dediff) was defined as MITF ${ }^{\text {low }}$ EGFR $^{\text {high }}$ or MITF $^{\text {low }}$ NGFR $^{\text {high }}$ and based on threshold values from ROC curves for each individual protein. d, Proportions of patients in the ZH adjuvant anti-PD-1 cohort with a dedifferentiated phenotype in the DF and Rel groups. e, Progression-free survival of patients with a dedifferentiated or differentiated melanoma phenotype. Statistical analysis was performed using the chi-square test (d) or Mantel-Cox log-rank test (e); $* \mathrm{P}<0.05, * * \mathrm{P}<0.01, * * * \mathrm{P}<0.001$. 


\section{Material and Methods}

324

325

326

327

328

329

330

331

332

333

334

335

336

337

338

340

341

342

\section{Ethical Approval}

All patients provided written informed consent. All study procedures were performed in accordance with the principles of the Declaration of Helsinki and applicable Swiss and Belgian laws and regulations, and approved by the Ethics Committee of Eastern Switzerland (BASEC Nr. 2016-00998, BASEC Nr. PB_2017-00494) and UZ Leuven Medical Ethical Committee (S62275).

\section{Patients}

In this prospective study, 27 patients with unresectable stage IV melanoma at Kantonsspital St. Gallen were included (SG cohort, see Extended Data Table 1 for patient information). Patients were treated with antibodies against the checkpoint inhibitor molecules PD-1 (pembrolizumab or nivolumab) either alone or in combination with anti-CTLA-4 (ipilimumab) and were included only if they had not received prior anti-PD-1 treatment. Tumor responses were evaluated by CT imaging after 8-12 weeks using RECIST $1.1^{27}$. According to these criteria, patients who achieved complete remission (CR) or partial remission (PR) were categorized as clinical responders (Rs), and those with stable disease (SD) or progressive disease (PD) were categorized as clinical nonresponders (NRs). For each patient, blood was drawn before start of immune checkpoint therapy. PBMCs were isolated using Ficoll-Paque gradient centrifugation and cryopreserved at $-150^{\circ} \mathrm{C}$. In addition, available pretreatment formalin-fixed and paraffin-embeded metastatic lesion biopsies taken for routine histopathological diagnosis from 19 patients in the SG cohort (10 Rs; 9 NRs) in proximity to the therapy start were used for IHC analysis.

In the second cohort, the Leuven cohort, metastatic lesion biopsies from 15 patients with locally advanced or metastatic melanoma (stage IIIB to stage IV) were collected as part of the noninterventional prospective study SPECIAL (prospective serial biopsy collection before and during immune checkpoint inhibitor therapy in patients with malignant melanoma) and used for 
single-cell RNA sequencing. Almost all patients $(\mathrm{N}=14)$ were treatment naïve. Details of the 348 samples are summarized in Extended Data Table 2.

349 A validation cohort of 30 stage III melanoma patients at the University Hospital Zurich who 350 received anti-PD-1 (pembrolizumab or nivolumab) as adjuvant therapy was used to confirm our 351 findings (ZH cohort, see Extended Data Table 3 for patient information). Response to therapy was 352 assessed at the first PET-CT scan (within 3 months after start of therapy) using RECIST 1.1 353 criteria $^{27}$, and patients were classified as either disease-free (DF) or experiencing a relapse (Rel) during the observation (up to 1 year after treatment cessation). Available pretreatment formalinfixed and paraffin-embedded tumor biopsies taken for routine histopathological diagnosis from all the patients in proximity to the therapy start were used for IHC analysis (19 DF; 11 Rel).

\section{T cell stimulation assays and flow cytometry}

358 For each patient, blood was drawn before the start of therapy, and PBMCs were isolated using

359 Ficoll-Paque density gradient centrifugation and cryopreserved at $-150^{\circ} \mathrm{C}$. To assess $\mathrm{T}$ cell 360 responses, PBMCs were thawed and cultured at $37^{\circ} \mathrm{C}$ and $5 \% \mathrm{CO}_{2}$ in $\mathrm{RPMI} 1640$ medium 361 containing $8 \%$ human serum (Biowest, catalog number S4190-100), 1\% penicillin-streptomycin, $3621 \%$ L-glutamine, $1 \%$ nonessential amino acids, $1 \%$ sodium pyruvate, $0.1 \mathrm{mg} / \mathrm{mL}$ kanamycin and $3630.1 \% \beta$-mercaptoethanol. PBMCs were seeded in 24 -well plates at a density of $0.5-1 \times 10^{6}$ cells per 364 well and stimulated with 15 -mer peptide pools ( $2 \mu \mathrm{g} / \mathrm{mL}$ peptide) with an 11-amino-acid overlap 365 for the following MDAs and CTAs according to the manufacturer's instructions: gp100, TYR, 366 TRP-2, MART-1, MAGE-A1, MAGE-A3, MAGE-A4, and NY-ESO-1 (JPT, Melanoma PepMix). 367 Every second day, half of the culture medium was replaced with fresh medium supplemented with $36815000 \mathrm{IU} / \mathrm{mL}$ recombinant human IL-2 (ProLeukin, Roche). After 10 days, the cells were washed 369 and restimulated for 6 hours with $2 \mu \mathrm{g} / \mathrm{mL}$ cognate peptide pools in the presence of $10 \mu \mathrm{g} / \mathrm{mL}$ 370 brefeldin-A (Sigma), followed by staining for flow cytometry. PBMCs were stained with Aqua 371 (BV510, BD, dilution 1:1000) according to the manufacturer's instructions to exclude dead cells 
and with antibodies against CD8 (PerCP, clone SK1, dilution 1:100), CD3 (APC-Cy7, clone

373 OKT3, dilution 1:100), CD45RA (BV711, clone HI100, dilution 1:400), CD4 (PE/Cy, clone

374 OTK4, dilution 1:100) in FACS buffer (1xPBS, 2\% FCS, 5 mM EDTA, 0.1\% Sodium azide) for

$37520 \mathrm{~min}$ at $4^{\circ} \mathrm{C}$. For intracellular staining, cells were fixed and permeabilized using the BD

376 Cytofix/Cytoperm (BD Biosciences) and staining with TNF- $\alpha$ (APC, clone Mab11b, dilution

377 1:100) and IFN- $\gamma(\mathrm{PE}$, clone B27, dilution 1:100) was done in permeabilization buffer (1xPBS,

$3782 \%$ FCS, $5 \mathrm{mM}$ EDTA, $0.1 \%$ Saponin, $0.1 \%$ Sodium azide) for $40-60 \mathrm{~min}$ at $4{ }^{\circ} \mathrm{C}$. Samples were

379 run on an LSR Fortessa, and flow cytometry data were analyzed with FlowJo software (TreeStar).

380 Antigen-specific $\mathrm{CD}^{+} \mathrm{T}$ cell responses were identified by subtracting the frequency of IFN- $\gamma^{+}$

381 and TNF- $\alpha^{+} \mathrm{CD}^{+}$T cells from the unstimulated control and using a cutoff value of $1 \%$ IFN- $\gamma^{+}$or

382 TNF- $\alpha^{+}$cells.

383 Immunohistochemistry

384 Available baseline tissue samples taken for diagnostic histological examination were fixed in

385 formalin and embedded in paraffin in the Pathology Department of KSSG using standard 386 processing protocols. Four-micron-thick serial sections were cut using a Leica RM2255 rotary

387 microtome (Leica Microsystems, CH). Single-epitope enzymatic immunohistochemistry on FFPE 388 tissues was performed on serial sections to assess the expression of TYR, MART-1, gp100, MITF,

389 EGFR, and NGFR within the tumors (immunohistochemical protocols for each antigen are 390 described in the subsequent sections). Morphometric analysis was carried out on whole-slide scans 391 acquired with a Panoramic 250 Flash III digital slide scanner (3D Histech, CH). Quantitative 392 morphometry was performed using the QuPath v0.1.2 software platform for whole-slide image 393 analysis (Edinburgh, UK) ${ }^{42}$ in order to determine the percentage of tumor cells expressing the 394 antigens interrogated by immunohistochemistry. 
398 FFPE tissues were sliced at a thickness of $4 \mu \mathrm{m}$ using a rotary microtome and placed on poly-L399 lysine-coated slides. The slides were dewaxed in xylene, rehydrated, subjected to heat-induced 400 epitope retrieval (HIER) in a microwave oven using a pH 9 target retrieval solution (catalog 401 number S2367, Dako, DK) for TYR and a pH 6 target retrieval solution (catalog number S1699, 402 Dako, DK) for 40 minutes, and allowed to cool to room temperature. Endogenous alkaline 403 phosphatase activity was blocked using the dual endogenous blocking solution from the EnVisionTM G/2 Doublestain System, Rabbit/Mouse (DAB+/Permanent Red) (catalog number K536111-2, Dako, DK) for 10 minutes at room temperature, followed by an avidin/biotin blocking step for 30 minutes at room temperature (catalog number SP-2001, Vector Laboratories, CA, USA), a 30-minute incubation with $3 \%$ skim milk at room temperature, and an overnight incubation at $4{ }^{\circ} \mathrm{C}$ with a mouse monoclonal anti-human TYR primary antibody (catalog number M3623, Dako, DK; dilution 1:50) and mouse monoclonal anti-human MITF primary antibody (catalog number M3621, Dako, DK; dilution 1:25). The next day, the slides were incubated for 1 411 hour at room temperature with a biotinylated anti-mouse secondary antibody (catalog number 115412 065-062, Jackson ImmunoResearch, Cambridge, UK; dilution 1:500), followed by another 1-hour 413 incubation at room temperature with a streptavidin-AP conjugate (ref. 434322, Invitrogen, IL, 414 USA; dilution 1:100). The signal was visualized using the Warp Red chromogen kit (catalog 415 number WR 806, Biocare Medical, CA, USA). The slides were dehydrated with alcohol, cleared 416 with xylene and mounted with Pertex (catalog number 41-4011-00, Medite, DE) after hematoxylin 417 counterstaining (catalog number 8947.1, Roth, DE).

\section{MART-1, gp100, and EGFR enzymatic immunohistochemistry}

419 Single-epitope enzymatic immunohistochemistry for MART-1, gp100, and EGFR in FFPE tissues 420 was performed on serial sections using a Leica BOND MAX III automated immunostainer (Leica, $421 \mathrm{CH}$ ) and the following antibodies according to the manufacturer's instructions: monoclonal mouse 
anti-human Melan A (Dako, catalog number M7196, clone A103, dilution 1:150, HIER - pH 9/20 minutes $/ 95^{\circ} \mathrm{C}$, incubation for $15 \mathrm{~min}$ ), monoclonal mouse anti-human Melanosome (gp100) (Dako, catalog number M0634, clone HMB-45, dilution 1:100, HIER - pH 6/20 minutes/100C, incubation for 30 minutes), and monoclonal mouse anti-human EGFR (Leica Biosystems, catalog number NCL-EGFR-384, dilution 1:60, HIER - $\mathrm{pH} 9 / 30$ minutes $/ 95^{\circ} \mathrm{C}$, incubation for 60 minutes). The signal was visualized using the Leica Bond Polymer Refine Detection Kit (catalog number DS9800, Leica Biosystems, CH) and the AEC chromogen (catalog number K050110, Diagnostic BioSystems, $\mathrm{CH})$.

\section{NGFR enzymatic immunohistochemistry}

FFPE tissues were sliced at a thickness of $4 \mu \mathrm{m}$ using a rotary microtome and placed on poly-Llysine-coated slides. The slides were dewaxed in xylene, rehydrated, subjected to HIER using a pH 9 target retrieval solution (catalog number S2367, Dako, DK) for 20 minutes in a microwave oven, and allowed to cool to room temperature. Endogenous horseradish peroxidase activity was blocked by a 10-minute incubation at room temperature with $3 \%$ hydrogen peroxide solution (catalog number 160-0-029, Laboratorium Dr. G. Bichsel AG, CH), followed by an avidin/biotin blocking step (catalog number SP-2001, Vector Laboratories, CA, USA) for 30 minutes at room temperature, a 30-minute incubation with $3 \%$ skim milk at room temperature, and an overnight incubation at $4{ }^{\circ} \mathrm{C}$ with a rabbit monoclonal anti-human p75 NGF receptor primary antibody (catalog number ab221212, Abcam, USA; dilution 1:100). The next day slides were incubated for 30 minutes at room temperature with an anti-rabbit biotinylated secondary antibody (catalog 442 number D30-1, GBI Labs, Bothell, WA, USA; dilution 1:200), followed by another 30-minute 443 incubation at room temperature with HRP-conjugated streptavidin (catalog number D30-1, GBI 444 Labs, Bothell, WA, USA; dilution 1:200). The signal was visualized using a Vector VIP 445 peroxidase substrate kit (catalog number SK-4600, Vector Laboratories, CA, USA). The slides 446 were dehydrated with alcohol, cleared with xylene and mounted with Pertex (catalog number 41- 
4011-00, Medite, DE) after methyl green counterstaining (catalog number H-3402, Vector

448 Laboratories, CA, USA).

\section{$449 \quad$ Image analysis}

450 Quantitative morphometry was performed on all stained slides using the QuPath v0.1.2 software 451 platform for whole-slide image analysis (Edinburgh, UK) ${ }^{42}$. Whole-slide scans acquired with a 452 Panoramic 250 Flash III digital slide scanner (3D Histech, $\mathrm{CH}$ ) were individually loaded into the software. For each staining vector (i.e., color), estimates of the background staining were applied to maximize differences in expression in QuPath by selecting a region of interest containing an area of background along with examples of strong nuclear counterstaining and chromogen staining and applying QuPath's “Estimate stain vectors" command to identify stain vectors within this region. The "Cell detection" command was then used to identify cells across the entire tissue section based upon nuclear identification. The full extent of each cell (area occupied by the cell) was estimated based upon measurements of intensity and morphology, including nuclear area, circularity, staining intensity for counterstaining and chromogen staining, and nucleus/cell area ratio. A two-way boosted decision tree classifier was then interactively trained to distinguish tumor

462 cells from all other detected artefacts (comprising nontumor epithelial cells, immune cells, stromal cells, necrosis, and any artefacts misidentified as cells). Cells were classified as positive or negative based upon a single-intensity threshold applied to the maximum optical density of the detected chromogen within the nucleus or cytoplasm of the cell depending on the expression

466 pattern (the threshold was adjusted for a random region of interest containing both negative and 467 highly positive tumor cells until all the cells defined as positive by the software matched those considered positive by the evaluator). Summary scores were generated as the percentage of cells classified as positive after "other" detections were removed. 


\section{Library construction and sequencing}

473 Metastatic lesions of 15 patients with locally advanced or metastatic melanoma (stage IIIB to stage

474 IV , 14 treatment-naive) were collected and scRNA-seq analysis was performed. Libraries for 475 scRNA-seq were created using the $10 \mathrm{X}$ Genomics Chromium platform according to the 476 manufacturer's instructions. Library construction was performed with the Chromium Single Cell 477 3' GEM, Library \& Gel Bead Kit v3 ( $\mathrm{n}=16$; 10x Genomics, catalog number 1000092) or the 478 Chromium Single Cell A Chip Kit and 5' Library \& Gel Bead Kit (10x Genomics, catalog number 479 1000014). We opted for high target recovery (median 5000, range 5000-10000), keeping within 480 the range of optimal input concentration per target recovery, as recommended by the manufacturer. 481 In brief, cells were partitioned into gel bead-in-emulsions (GEMs) at limiting dilutions, where lysis 482 and reverse transcription occurred, yielding uniquely barcoded full-length cDNA from 483 polyadenylated mRNA. GEMs were subsequently broken, and the pooled fraction was amplified 484 and subjected to fragmentation, end repair and adaptor ligation of size-selected fractions.

\section{scRNA-seq data acquisition and preprocessing}

486 All libraries were sequenced on an Illumina NextSeq, HiSeq4000 or NovaSeq6000 until sufficient 487 saturation was reached $(73.8 \%$ on average). After quality control, the raw sequencing reads were 488 aligned to the human reference genome v. GRCh38, followed by processing with CellRanger (10x 489 Genomics, v2.0) to obtain feature-barcode matrices. Raw count matrices were analyzed using the 490 R package Seurat v. 3.1.34 ${ }^{43}$. The matrices were filtered for genes expressed in at least 10 cells 491 and cells that expressed at least 1000 genes. The SCTransform function in Seurat was applied to 492 each Seurat object separately for data normalization and transformation, followed by the 493 application of DoubletFinder v. $2 \cdot 0 \cdot 28^{44}$ to each Seurat object separately to remove doublets 494 (assuming that the doublet rate in each sample was 3.9\%). Next, all the Seurat objects were merged 495 and filtered by removing cell barcodes with <1000 expressed genes, >7500 expressed genes or $496>30 \%$ reads mapping to mitochondrial genes. The SCTransform function from the Seurat package 
was applied to regress out the mitochondrial read percentage per cell. Subsequently, data integration was performed using the R package Harmony v. $1.04^{45}$. After data normalization and integration, cell cycle scoring was performed per cell, data were filtered for singlets, and SCTransform was again applied to regress out the mitochondrial read percentage and cell cycle 501 scores, followed by data integration of this subset as described above.

\section{2 \\ Malignant cell identification and CNV inference}

To identify malignant cells, two gene sets specific for melanoma were used; these gene sets were acquired from Tirosh et al. ${ }^{46}$ (Extended Data Table 4) and Rambow et al. ${ }^{34}$ (Extended Data Table 5). The gene activity of these two gene sets was scored using the R package AUCell v.1.6.14 ${ }^{47}$. Moreover, we calculated the copy number variation (CNV) based on scRNA-seq data by using the R package HoneyBADGER v. $0.14^{48}$. The input for HoneyBADGER was the count matrix from the "RNA" assay from the integrated Seurat object of all cells. The reference for normal cells was immune cells, which were also identified by AUCell using the immune gene set from Jerby-Arnon et al. ${ }^{49}$. The mean CNV score was calculated as follows:

$$
\mathrm{CNV} \text { score }=\frac{\sum_{i}\left|G_{\mathrm{cnv}, i}\right|}{n}
$$

where $\mathrm{G}=$ gene and $i=$ cell.

514 Next, the mean CNV score was correlated with Tirosh et al. ${ }^{46}$ and Rambow et al. ${ }^{34}$ gene set activity. 515 Based on the strength of the correlation, the gene set was used for further data analysis for 516 malignant cells. The subsets of malignant cells were subjected to SCTransform (regressing out the mitochondrial read percentage and cell cycle scores) and Harmony integration followed by Seurat

518 clustering. The number of dimensions for clustering was chosen based on Harmony embedding 519 clustering; the cutoff was driven by the identification of clear variation in embeddings across the 520 cells. The activity of different gene expression sets was quantified using AUCell ${ }^{47}$. 


\section{Statistical analysis}

523 Statistical analysis was performed using GraphPad 7.0. Unless otherwise specified, graphs depict

524 the mean or median \pm SD or SEM. Differences between two groups were evaluated using the 525 unpaired Mann-Whitney test or chi-square test. Kaplan-Meier survival curves were assessed to 526 calculate progression-free survival using the log-rank test. The results were considered statistically 527 significant when $\mathrm{P}<0.05(*), \mathrm{P}<0.01(* *)$ or $\mathrm{P}<0.001(* * *)$. Heatmaps summarizing IFN- $\gamma^{+}$or $528 \mathrm{TNF}-\alpha^{+} \mathrm{CD}^{+} \mathrm{T}$ cell responses or IHC signals as percentages of tissue area were used. Receiver 529 operator characteristic (ROC) curves were used to determine the thresholds identifying high vs 530 low expression in IHC analysis. 


\section{References}

5331 Dunn, G. P., Bruce, A. T., Ikeda, H., Old, L. J. \& Schreiber, R. D. Cancer

534

535

536

537

538

539

540

541

542

543

544

545

546

547

548

549

550

551

552

553

554

555

556

557

558

559

560

561

562

563

564

565

566

567

568

569

570

571

572

573

574

575

576

577

578

579 immunoediting: from immunosurveillance to tumor escape. Nat Immunol 3, 991-998, doi:10.1038/ni1102-991 (2002).

2 Schreiber, R. D., Old, L. J. \& Smyth, M. J. Cancer immunoediting: integrating immunity's roles in cancer suppression and promotion. Science 331, 1565-1570, doi:10.1126/science.1203486 (2011).

3 Knuth, A., Wolfel, T., Klehmann, E., Boon, T. \& Meyer zum Buschenfelde, K. H. Cytolytic T-cell clones against an autologous human melanoma: specificity study and definition of three antigens by immunoselection. Proc Natl Acad Sci U S A 86, 28042808 (1989).

4 Kawakami, Y. et al. Recognition of shared melanoma antigens in association with major HLA-A alleles by tumor infiltrating T lymphocytes from 123 patients with melanoma. $J$ Immunother 23, 17-27, doi:10.1097/00002371-200001000-00004 (2000).

5 Germeau, C. et al. High frequency of antitumor T cells in the blood of melanoma patients before and after vaccination with tumor antigens. J Exp Med 201, 241-248, doi:10.1084/jem.20041379 (2005).

6 Coulie, P. G. et al. Precursor frequency analysis of human cytolytic T lymphocytes directed against autologous melanoma cells. Int J Cancer 50, 289-297, doi:10.1002/ijc.2910500220 (1992).

7 Tumeh, P. C. et al. PD-1 blockade induces responses by inhibiting adaptive immune resistance. Nature 515, 568-571, doi:10.1038/nature13954 (2014).

8 Herbst, R. S. et al. Predictive correlates of response to the anti-PD-L1 antibody MPDL3280A in cancer patients. Nature 515, 563-567, doi:10.1038/nature14011 (2014).

9 Burnet, F. M. Immunological surveillance in neoplasia. Transplant Rev 7, 3-25 (1971).

10 Landsberg, J. et al. Melanomas resist T-cell therapy through inflammation-induced reversible dedifferentiation. Nature 490, 412-416, doi:10.1038/nature11538 (2012).

11 Effern, M. et al. Adoptive T Cell Therapy Targeting Different Gene Products Reveals Diverse and Context-Dependent Immune Evasion in Melanoma. Immunity 53, 564-580 e569, doi:10.1016/j.immuni.2020.07.007 (2020).

12 Holzel, M., Bovier, A. \& Tuting, T. Plasticity of tumour and immune cells: a source of heterogeneity and a cause for therapy resistance? Nat Rev Cancer 13, 365-376, doi:10.1038/nrc3498 (2013).

13 Holzel, M. \& Tuting, T. Inflammation-Induced Plasticity in Melanoma Therapy and Metastasis. Trends Immunol 37, 364-374, doi:10.1016/j.it.2016.03.009 (2016).

14 Boiko, A. D. et al. Human melanoma-initiating cells express neural crest nerve growth factor receptor CD271. Nature 466, 133-137, doi:10.1038/nature09161 (2010).

15 Jager, E. et al. Immunoselection in vivo: independent loss of MHC class I and melanocyte differentiation antigen expression in metastatic melanoma. Int J Cancer 71, 142-147, doi:10.1002/(sici)1097-0215(19970410)71:2<142::aid-ijc3>3.0.co;2-0 (1997).

16 Perez-Guijarro, E. et al. Multimodel preclinical platform predicts clinical response of melanoma to immunotherapy. Nat Med 26, 781-791, doi:10.1038/s41591-020-0818-3 (2020).

17 Lee, J. H. et al. Transcriptional downregulation of MHC class I and melanoma dedifferentiation in resistance to PD-1 inhibition. Nat Commun 11, 1897, doi:10.1038/s41467-020-15726-7 (2020).

18 Mehta, A. et al. Immunotherapy Resistance by Inflammation-Induced Dedifferentiation. Cancer Discov 8, 935-943, doi:10.1158/2159-8290.CD-17-1178 (2018). 
19 Kvistborg, P. et al. Anti-CTLA-4 therapy broadens the melanoma-reactive CD8+ T cell

581

582

583

584

585

586

587

588

589

590

591

592

593

594

595

596

597

598

599

600

601

602

603

604

605

606

607

608

609

610

611

612

613

614

615

616

617

618

619

620

621

622

623

624

625

626

627

20 Coulie, P. G., Van den Eynde, B. J., van der Bruggen, P. \& Boon, T. Tumour antigens recognized by T lymphocytes: at the core of cancer immunotherapy. Nat Rev Cancer 14, 135-146, doi:10.1038/nrc3670 (2014).

21 Chen, Y. T. et al. Identification of multiple cancer/testis antigens by allogeneic antibody screening of a melanoma cell line library. Proc Natl Acad Sci U S A 95, 6919-6923, doi:10.1073/pnas.95.12.6919 (1998).

22 Schumacher, T. N. \& Schreiber, R. D. Neoantigens in cancer immunotherapy. Science 348, 69-74, doi:10.1126/science.aaa4971 (2015).

23 Hua, C. et al. Association of Vitiligo With Tumor Response in Patients With Metastatic Melanoma Treated With Pembrolizumab. JAMA Dermatol 152, 45-51, doi:10.1001/jamadermatol.2015.2707 (2016).

24 Yeh, S. et al. Ocular and systemic autoimmunity after successful tumor-infiltrating lymphocyte immunotherapy for recurrent, metastatic melanoma. Ophthalmology 116, 981-989 e981, doi:10.1016/j.ophtha.2008.12.004 (2009).

25 Gogas, H. et al. Prognostic significance of autoimmunity during treatment of melanoma with interferon. N Engl J Med 354, 709-718, doi:10.1056/NEJMoa053007 (2006).

26 Larkin, J. et al. Combined Nivolumab and Ipilimumab or Monotherapy in Untreated Melanoma. $N$ Engl J Med 373, 23-34, doi:10.1056/NEJMoa1504030 (2015).

27 Eisenhauer, E. A. et al. New response evaluation criteria in solid tumours: revised RECIST guideline (version 1.1). Eur J Cancer 45, 228-247, doi:10.1016/j.ejca.2008.10.026 (2009).

28 Wolchok, J. D. et al. Guidelines for the evaluation of immune therapy activity in solid tumors: immune-related response criteria. Clin Cancer Res 15, 7412-7420, doi:10.1158/1078-0432.CCR-09-1624 (2009).

29 Robert, C. et al. Pembrolizumab versus Ipilimumab in Advanced Melanoma. N Engl J Med 372, 2521-2532, doi:10.1056/NEJMoa1503093 (2015).

30 Matsushita, H. et al. Cancer exome analysis reveals a T-cell-dependent mechanism of cancer immunoediting. Nature 482, 400-404, doi:10.1038/nature10755 (2012).

31 Rosenberg, S. A. \& Restifo, N. P. Adoptive cell transfer as personalized immunotherapy for human cancer. Science 348, 62-68, doi:10.1126/science.aaa4967 (2015).

32 Rosenberg, S. A. et al. Durable complete responses in heavily pretreated patients with metastatic melanoma using T-cell transfer immunotherapy. Clin Cancer Res 17, 45504557, doi:10.1158/1078-0432.CCR-11-0116 (2011).

33 Tsoi, J. et al. Multi-stage Differentiation Defines Melanoma Subtypes with Differential Vulnerability to Drug-Induced Iron-Dependent Oxidative Stress. Cancer Cell 33, 890904 e895, doi:10.1016/j.ccell.2018.03.017 (2018).

34 Rambow, F. et al. New Functional Signatures for Understanding Melanoma Biology from Tumor Cell Lineage-Specific Analysis. Cell Rep 13, 840-853, doi:10.1016/j.celrep.2015.09.037 (2015).

35 Hoek, K. S. et al. In vivo switching of human melanoma cells between proliferative and invasive states. Cancer Res 68, 650-656, doi:10.1158/0008-5472.CAN-07-2491 (2008).

36 Verfaillie, A. et al. Decoding the regulatory landscape of melanoma reveals TEADS as regulators of the invasive cell state. Nat Commun 6, 6683, doi:10.1038/ncomms7683 (2015).

37 Sun, C. et al. Reversible and adaptive resistance to BRAF(V600E) inhibition in melanoma. Nature 508, 118-122, doi:10.1038/nature13121 (2014). 
62838 Kanik, A. B., Yaar, M. \& Bhawan, J. p75 nerve growth factor receptor staining helps identify desmoplastic and neurotropic melanoma. J Cutan Pathol 23, 205-210, doi:10.1111/j.1600-0560.1996.tb01468.x (1996).

632

633

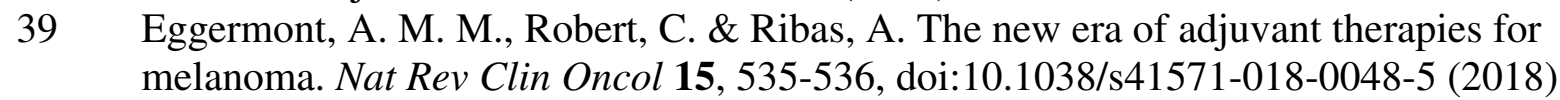

40 Eggermont, A. M. M., Robert, C. \& Suciu, S. Adjuvant Pembrolizumab in Resected

634

635 Stage III Melanoma. N Engl J Med 379, 593-595, doi:10.1056/NEJMc1807505 (2018).

636

637

638

639

640

641

642

643

644

645

646

647

648 Huang, A. C. et al. T-cell invigoration to tumour burden ratio associated with anti-PD-1

42 Bankhead, P. et al. QuPath: Open source software for digital pathology image analysis. Sci Rep 7, 16878, doi:10.1038/s41598-017-17204-5 (2017).

43 Stuart, T. et al. Comprehensive Integration of Single-Cell Data. Cell 177, 1888-1902 e1821, doi:10.1016/j.cell.2019.05.031 (2019).

44 McGinnis, C. S., Murrow, L. M. \& Gartner, Z. J. DoubletFinder: Doublet Detection in Single-Cell RNA Sequencing Data Using Artificial Nearest Neighbors. Cell Syst 8, 329337 e324, doi:10.1016/j.cels.2019.03.003 (2019).

45 Korsunsky, I. et al. Fast, sensitive and accurate integration of single-cell data with Harmony. Nat Methods 16, 1289-1296, doi:10.1038/s41592-019-0619-0 (2019).

46 Tirosh, I. et al. Dissecting the multicellular ecosystem of metastatic melanoma by singlecell RNA-seq. Science 352, 189-196, doi:10.1126/science.aad0501 (2016).

47 Aibar, S. et al. SCENIC: single-cell regulatory network inference and clustering. Nat

649 Methods 14, 1083-1086, doi:10.1038/nmeth.4463 (2017).

650

48 Fan, J. et al. Linking transcriptional and genetic tumor heterogeneity through allele

651

652

653 analysis of single-cell RNA-seq data. Genome Res 28, 1217-1227, doi:10.1101/gr.228080.117 (2018).

49 Jerby-Arnon, L. et al. A Cancer Cell Program Promotes T Cell Exclusion and Resistance to Checkpoint Blockade. Cell 175, 984-997 e924, doi:10.1016/j.cell.2018.09.006 (2018).

655

656 
658 The authors thank Caroline Dietrich and Martina Kurz from the Clinical Trials Unit, Kantonsspital 659 St.Gallen, for the excellent management of the St.Gallen patient cohort. The authors also thank 660 Simone Golling, Institute of Pathology at the Kantonsspital St.Gallen, and Romina Zarbl, 661 University of Bonn, for providing excellent technical support. We also thank the NGS Core 662 Facility of the University Hospital Bonn. We appreciate the support of the University of Zurich 663 Research Priority Program (URPP). The manuscript was edited for proper English language, 664 grammar, punctuation, spelling, and overall style by one or more of the highly qualified native 665 English speaking editors at SNAS.

666

\section{Funding}

668 This study was funded in part by grant \#PP00P3_157448 from the Swiss National Science 669 Foundation and grant \#18A042 from the Novartis Foundation for Medical Biological Research 670 (both to L.F.). The study coordination of the St. Gallen patient cohort was supported by a grant 671 from the Forschungsförderung of the Kantonsspital St Gallen. M.H. was supported by the 672 Deutsche Forschungsgemeinschaft (DFG, German Research Foundation) under Germany's 673 Excellence Strategy - EXC2151 - 390873048. M.v.d.B was funded by the Swiss National Science 674 Foundation (CRSII5_177208 and 310030_175565) and the University of Zurich (University 675 Research Priority Program "Translational Cancer Research”).

\section{Author contributions}

678 L.F., S.S.R., O.T.P. designed the study, discussed data and wrote the paper; S.S.R., O.T.P., M.679 T.A. and S.N.F. performed experiments, analyzed and discussed data. J.P., E. L., F.R., G.B., O.B. 680 performed experiments and bioinformatics analyses and discussed data. F.B., O.H.A., M.-T.A., 681 S.D., R.N., M.F. managed the SG patient cohort and collected patient material. W.J. provided 
682 patient material and discussed data. J.M., R.D., and M.P.L. provided patient data and material for 683 the adjuvant Zurich Cohort. T.B., T.M., M.M. provided patient material. J.L., D.D., D.B., 684 M.v.d.B., A.C., C.G., M.P.L., B.L. discussed data. T.T., M.H., J.-C. M discussed data and provided 685 support to develop the manuscript.

686

687 Competing financial interests

688 L.F. discloses grants from the Swiss National Science Foundation, Swiss Cancer League, Hookipa 689 Pharma, and Novartis Foundation as well as advisory roles for Novartis, Sanofi and Bristol-Myers 690 Squibb. T.B. has a research agreement with Bristol-Myers Squibb.

691

692 Data and materials availability

693 scRNA-seq data will be available. Further information and requests for resources and reagents 694 should be directed to and will be fulfilled by the lead investigator, Lukas Flatz 695 (lukas.flatz@med.uni-tuebingen.de).

696 


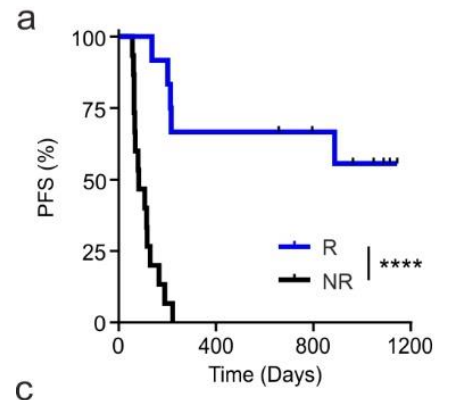

b
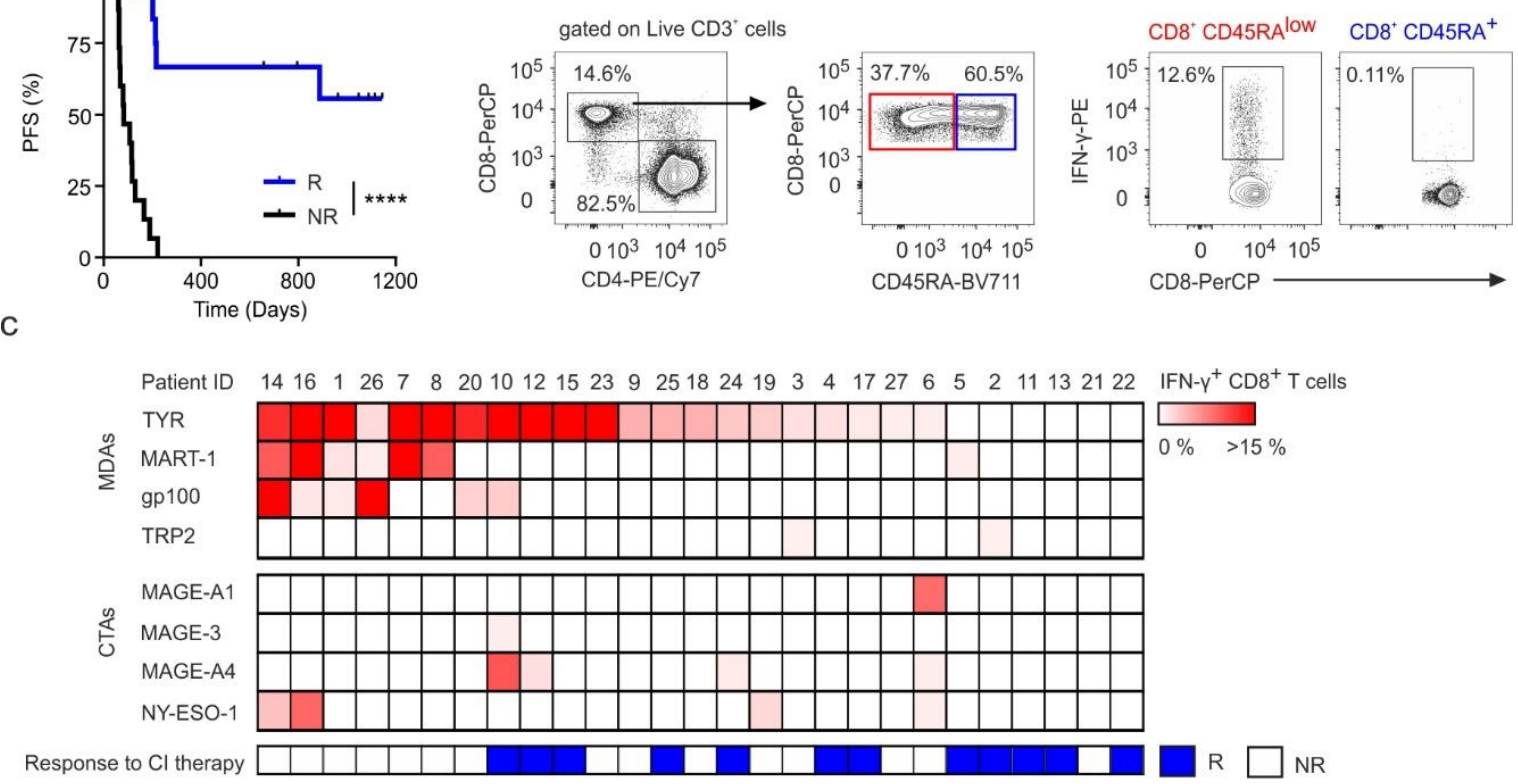

d

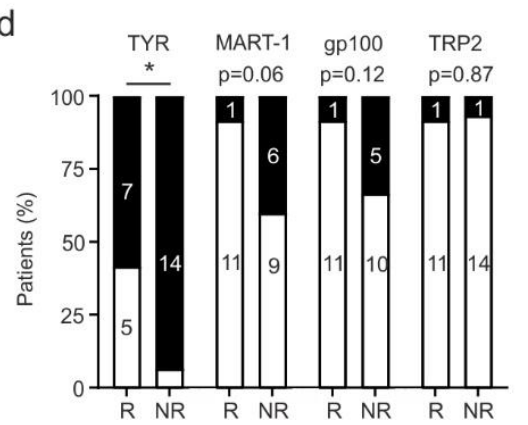

e

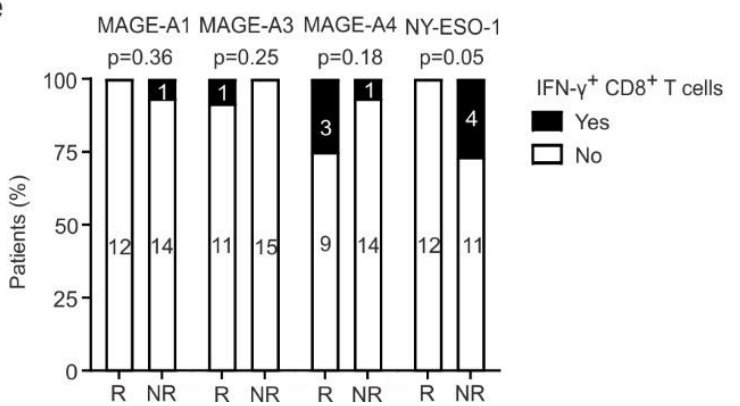

Extended Data Figure 1. Pre-existing MDA- and CTA-specific IFN- $\gamma^{+} \mathrm{CD8}^{+} \mathrm{T}$ cells in melanoma patients receiving checkpoint inhibitor therapy. a, Progression-free survival of patients with stage IV melanoma in the St. Gallen (SG) cohort. Clinical responders (R; complete or partial response; $n=12$ ) and nonresponders (NR; progressive disease; $\mathrm{n}=15$ ) to checkpoint inhibitor therapy. $\mathbf{b}$-e, PBMCs were obtained from SG cohort patients prior to checkpoint inhibitor therapy and stimulated in vitro with overlapping 15mer peptide pools specific for the melanocyte differentiation antigens (MDAs) TYR, MART-1, gp100, and TRP2 and the cancer testis antigens (CTAs) MAGE-A1, MAGE-A3, MAGE-A4, and NY-ESO-1. b, The FACS gating strategy is shown. Cytokine-producing cells were detected within the antigen-experienced CD45RA ${ }^{\text {low }} \mathrm{CD}^{+} \mathrm{T}$ cell population. $\mathbf{c}$, Heatmaps summarizing the frequencies of IFN- $\gamma$-producing $\mathrm{CD}^{+}$ $\mathrm{T}$ cells for the indicated antigens. The color scale represents the frequency of IFN- $\gamma^{+} \mathrm{CD} 3^{+} \mathrm{CD} 8^{+}$ CD45RA ${ }^{\text {low }} \mathrm{T}$ cells. d-e, Proportions of $\mathrm{R}$ and NR with IFN- $\gamma^{+} \mathrm{CD} 8^{+} \mathrm{T}$ cell responses against MDAs (d) and CTAs (e) after checkpoint inhibitor therapy. Statistical analysis was performed using log-rank (a) and 
a

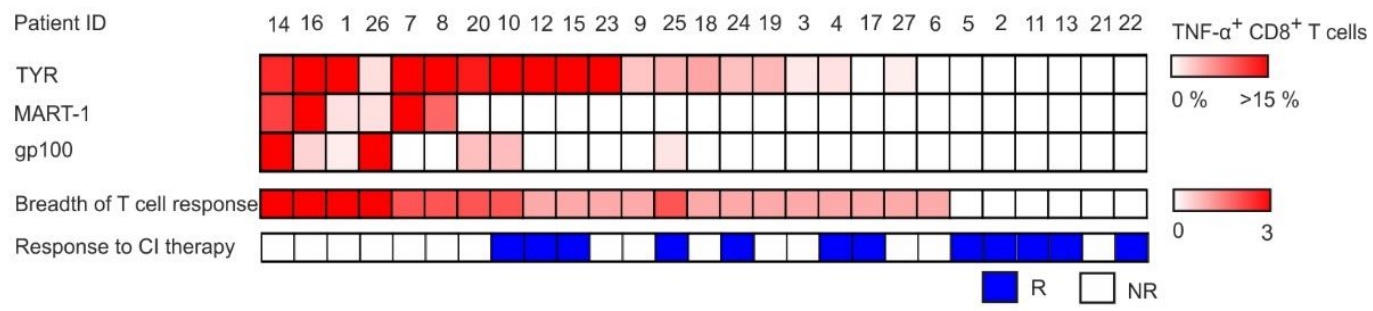

b

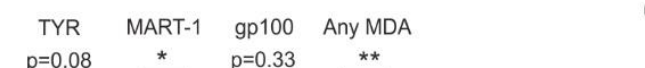

C

d
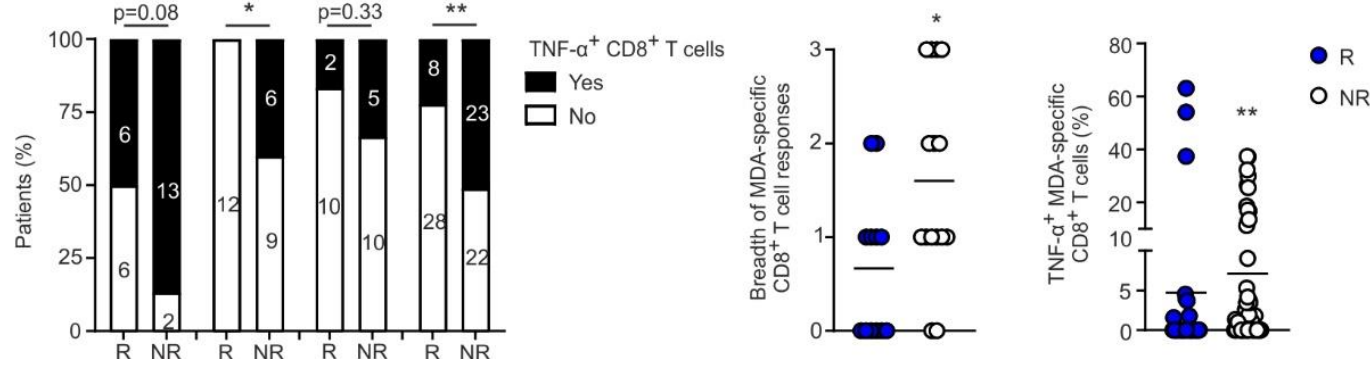

Extended Data Figure 2. Pre-existing MDA-specific $\mathbf{T N F}-\alpha^{+} \mathrm{CD8}^{+} \mathbf{T}$ cells in melanoma patients receiving checkpoint inhibitor therapy. a, Heatmaps summarizing TNF- $\alpha^{+} \mathrm{CD} 8^{+} \mathrm{T}$ cells in the SG cohort for the indicated antigens. The color scale represents the frequency of TNF- $\alpha^{+} \mathrm{CD} 3^{+} \mathrm{CD} 8^{+} \mathrm{CD} 45 \mathrm{RA}{ }^{\text {low }} \mathrm{T}$ cells. The breadth of the $\mathrm{CD}^{+} \mathrm{T}$ cell response was defined as the number of individual MDAs recognized, as indicated by the detection of $\mathrm{TNF}-\alpha^{+} \mathrm{CD}^{+} \mathrm{T}$ cell responses, ranging from 0 (none) to 3 (all). b, Proportions of $\mathrm{R}$ and $\mathrm{NR}$ with $\mathrm{TNF}-\alpha^{+} \mathrm{MDA}-$ specific $\mathrm{CD} 8^{+} \mathrm{T}$ cell responses after checkpoint inhibitor therapy. c, Summary of the breadth of MDA-specific $\mathrm{CD}^{+} \mathrm{T}$ cells in $\mathrm{R}$ and $\mathrm{NR}$ as shown for individual patients as shown in panel a. d, Frequencies of TNF- $\alpha^{+} \mathrm{CD}^{+} \mathrm{T}$ cells against any MDA (TYR, MART-1 or gp100) in R vs NR. Dots represent individual patients, and lines indicate mean values (c and d). Statistical analysis was performed using the chi-square test (b) and Mann-Whitney test (c and d); *P $<0.05$, **P $<$ $0.01, * * * \mathrm{P}<0.001$. 
a

b
C
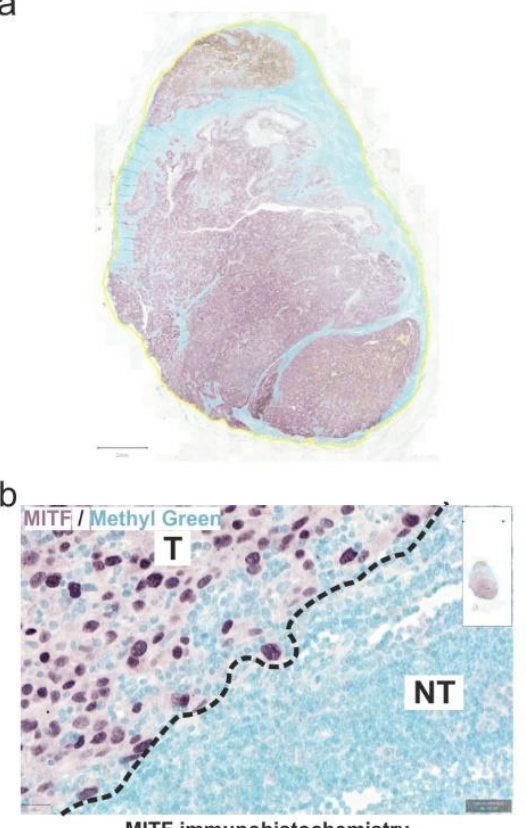

MITF immunohistochemistry
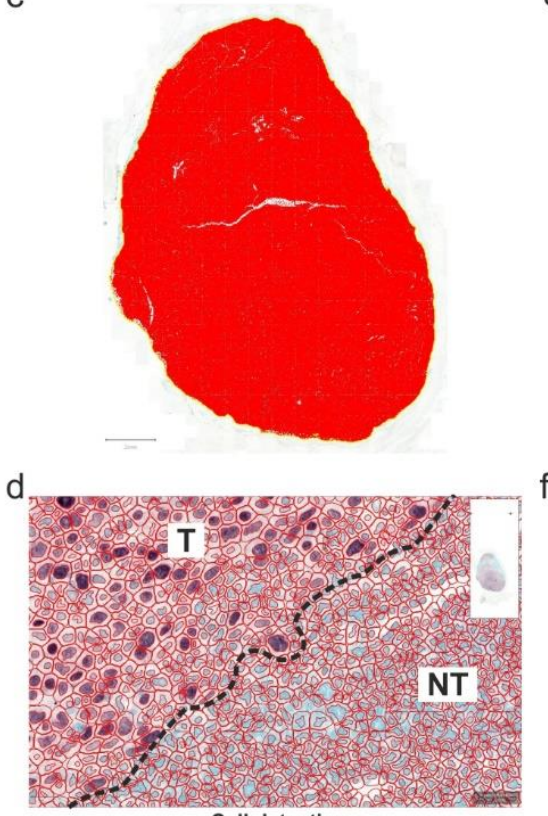

Cell detection
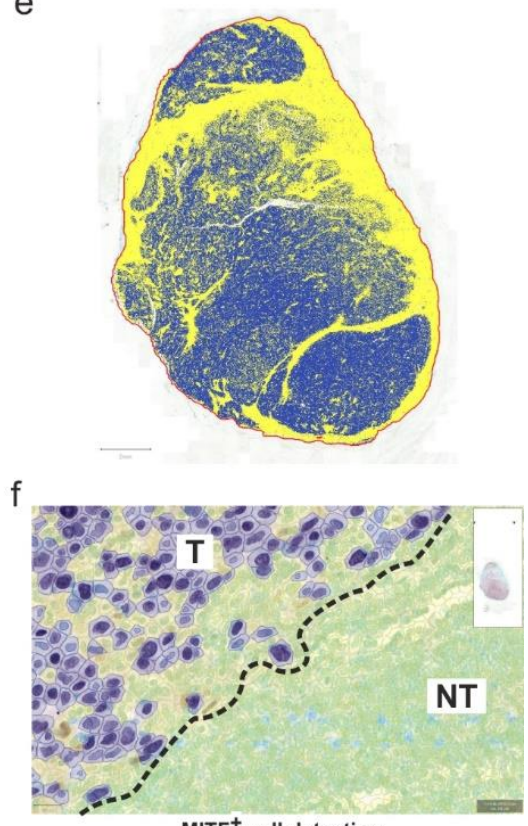

Extended Data Figure 3. Quantitative digital morphometry of enzymatic IHC using QuPath: image analysis strategy. a, Whole-slide scan of a lymph node metastasis stained for MITF (violet chromogen) with methyl green counterstaining. The selected region of interest for analysis is indicated (yellow outline). b, Detail showing MITF $^{+}$tumor cells (violet nuclei) and MITF- cells (NT); the dashed line separates tumor (T) and non-tumor (NT) areas (200× magnification). c, Cell identification (red mask) on the whole-slide scan using the "Cell detection command". d, Detailed results for cell identification (red cellular and nuclear contours) (200× magnification). e, Results of cell classification by training a two-way boosted decision tree classifier (blue mask - MITF ${ }^{+}$tumor cells; yellow mask - MITF- cells). f, Detail showing the results of cell classification (blue and yellow masks and contours identifying $\mathrm{MITF}^{+}$tumor cells and MITF- cells, respectively) (200× magnification). 
a

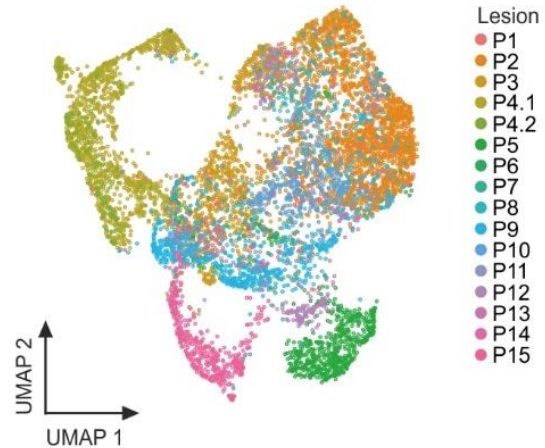

b

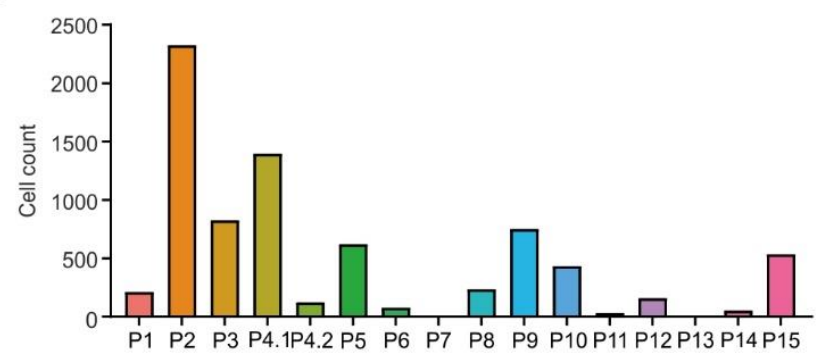

C

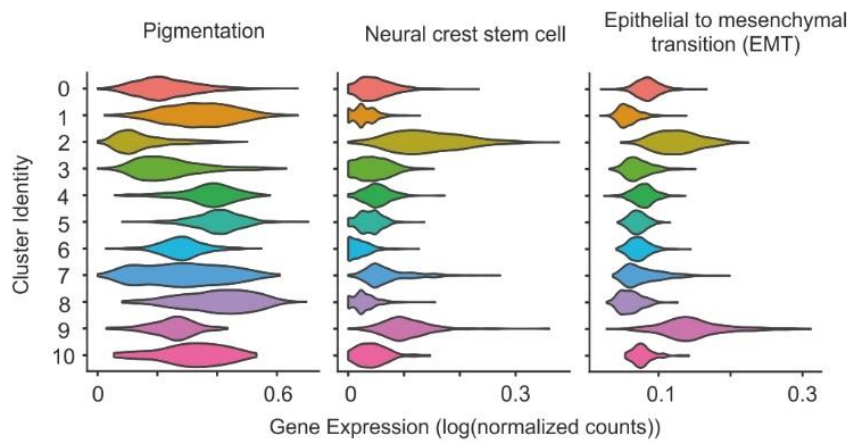

d
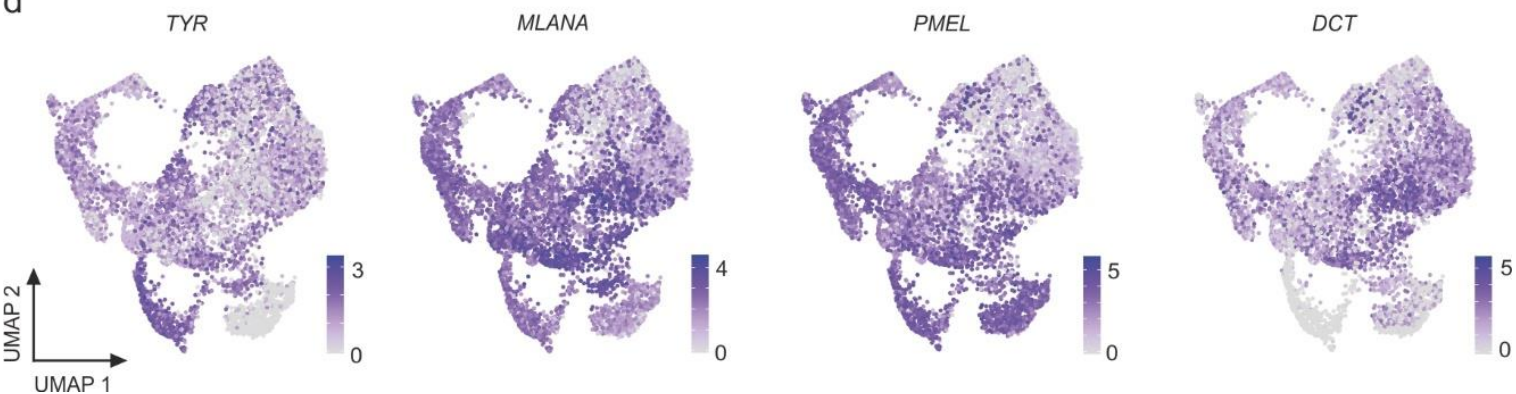

Extended Data Figure 4. Transcriptomic analysis revealing tumor cells with a dedifferentiated phenotype. a, Single-cell RNA sequencing of tumor cells from metastatic lesions obtained from 15 stage III and IV melanoma patients (Leuven cohort). UMAP plot displays individual lesion assignment to the assigned clusters as shown in Fig. 3a. b, Bar graph indicating the number of cells obtained from each lesion. c, Violin plots based on the Rambow et al. $(2015)^{34}$ gene signatures for pigmentation, neural crest stem cells (NCSCs) and epithelial-mesenchymal transition (EMT). Tumor cells in cluster 2, 3, 7 and 9 exhibit a dedifferentiated phenotype d, UMAP plots showing the gene expression of TYR, MLANA (MART-1), $P M E L$ (gp100) and DCT (TRP2). 


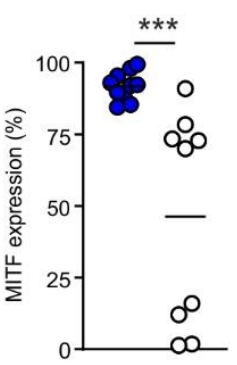

d

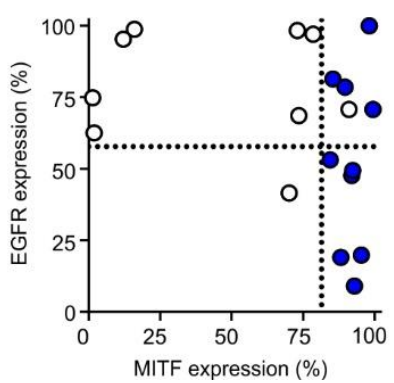

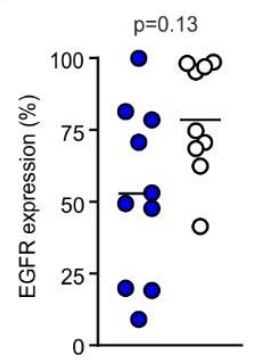

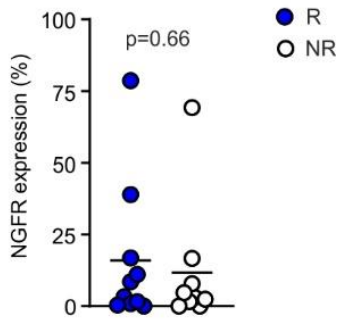

e

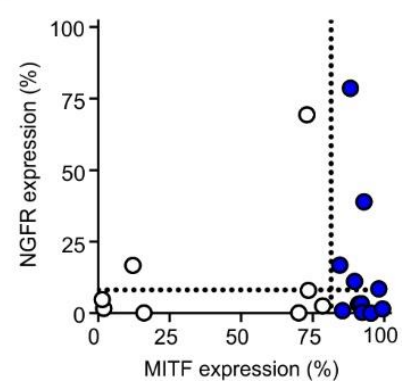

751
Extended Data Figure 5. The dedifferentiated tumor phenotype is associated with the clinical response to checkpoint inhibitor treatment. a-c, Frequencies of melanoma cells stained positive for MITF, EGFR or NGFR in responders (R) and nonresponders (NR). d-e, The dedifferentiated phenotype was defined as d, MITF ${ }^{\text {low }}$ EGFR $^{\text {high }}$ or e, MITF ${ }^{\text {low }}$ NGFR $^{\text {high }}$ as defined by threshold values from ROC curves for each individual protein (dotted lines). Dots represent individual patients (a-e) and lines indicate mean values $(\mathrm{a}-\mathrm{c})$. Statistical analysis was performed using the Mann-Whitney test; $* \mathrm{P}<0.05, * * \mathrm{P}<0.01$, $* * * \mathrm{P}<0.001$. 
a

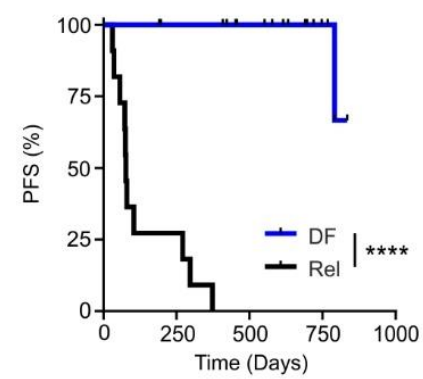

e

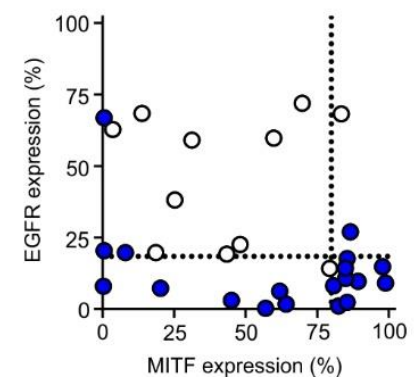

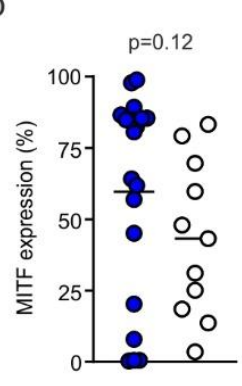

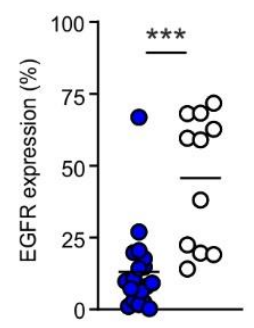

d

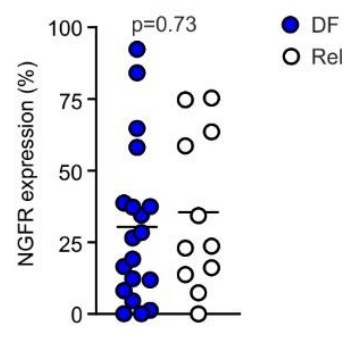

f

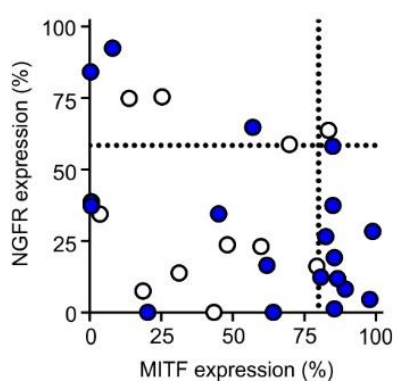

Extended Data Figure 6. A dedifferentiated tumor phenotype predicts the clinical response to adjuvant checkpoint inhibitor therapy. a, Progression-free survival of patients with stage III melanoma in the Zurich $(\mathrm{ZH})$ adjuvant anti-PD-1 cohort. Disease-Free patients (DF; $\mathrm{R}$; $\mathrm{n}=19$ ) and patients who experienced relapse (Rel; $n=11$ ) were determined at the first PET-CT scan within 3 months after start of therapy. e-f, Tumor biopsies were obtained before initiation of treatment. and frequencies of melanoma cells stained positive for MITF, EGFR or NGFR in the DF and Rel groups. e-f, The dedifferentiated phenotype was defined as e, MITF ${ }^{\text {low }}$ EGFR $^{\text {high }}$ or $\mathbf{f}$, MITF $^{\text {low }}$ NGFR $^{\text {high }}$ as defined by threshold values from ROC curves for each individual protein (dotted lines). Dots represent individual patients (b-f) and lines indicate mean values (b-d).Statistical analysis was performed using the log-rank (a) and Mann-Whitney tests $(\mathrm{d}-\mathrm{g}) ; * \mathrm{P}<0.05, * * \mathrm{P}<0.01, * * * \mathrm{P}<0.001$. 
Extended Data Table 1. Patient characteristics of the St.Gallen (SG) cohort

\begin{tabular}{|c|c|c|}
\hline Characteristic & $\begin{array}{c}\text { Responders } \\
(\mathrm{N}=12,44 \%)\end{array}$ & $\begin{array}{c}\text { Nonresponders } \\
(\mathrm{N}=15,56 \%)\end{array}$ \\
\hline \multicolumn{3}{|c|}{ Age at start of treatment - years } \\
\hline Median & 69 & 69 \\
\hline Range & $41-81$ & $47-86$ \\
\hline \multicolumn{3}{|l|}{ Sex - no. of patients (\%) } \\
\hline Female & $4(33.3)$ & $10(66.7)$ \\
\hline Male & $8(66.7)$ & $5(33.3)$ \\
\hline \multirow{2}{*}{\multicolumn{3}{|c|}{$\begin{array}{l}\text { Histological type (primary tumor) - } \\
\text { no. of patients }(\%)\end{array}$}} \\
\hline & & \\
\hline Superficial spreading & $5(41.7)$ & $7(46.6)$ \\
\hline Nodular & $3(25)$ & $1(6.7)$ \\
\hline Acral lentiginous & $1(8.3)$ & $0(0)$ \\
\hline Mucosal lentiginous & $0(0)$ & $1(6.7)$ \\
\hline Uveal & $1(8.3)$ & $3(20)$ \\
\hline Unknown primary & $2(16.7)$ & $3(20)$ \\
\hline \multicolumn{3}{|c|}{ BRAF mutation - no. of patients (\%) } \\
\hline No & $8(66.7)$ & $10(66.7)$ \\
\hline Yes & $4(33.3)$ & $5(33.3)$ \\
\hline \multicolumn{3}{|c|}{ CRP levels at start of therapy $-\mathrm{mg} / \mathrm{L}$} \\
\hline Median & 1 & 5 \\
\hline Range & $0-19$ & $0-114$ \\
\hline \multicolumn{3}{|l|}{ WBC at start of therapy - $\mathrm{g} / \mathrm{L}$} \\
\hline Median & 7.7 & 7.3 \\
\hline Range & 3-13.9 & $3.4-11$ \\
\hline \multicolumn{3}{|c|}{ Lymphocytes at start of therapy - $\mathrm{g} / \mathrm{L}$} \\
\hline Median & 1.6 & 1.2 \\
\hline Range & $0.4-2.9$ & $0.6-3$ \\
\hline \multicolumn{3}{|c|}{ Granulocytes at start of therapy $-\mathrm{g} / \mathrm{L}$} \\
\hline Median & 4.9 & 5.4 \\
\hline Range & $2.2-12.1$ & $1.8-8.7$ \\
\hline \multicolumn{3}{|l|}{$\begin{array}{l}\text { Checkpoint inhibitor therapy - } \\
\text { no. of patients }(\%)\end{array}$} \\
\hline pembrolizumab & $9(75)$ & $12(80)$ \\
\hline ipilimumab + nivolumab & $3(25)$ & $3(20)$ \\
\hline \multicolumn{3}{|c|}{ Progression-free survival - days* } \\
\hline Median & 481 & 107 \\
\hline Range & $137-878$ & $56-222$ \\
\hline \multicolumn{3}{|l|}{ Overall survival - days $\dagger$} \\
\hline Median & 615 & 371 \\
\hline Range & $256-931$ & $107-908$ \\
\hline
\end{tabular}

* Progression-free survival was calculated from the date of first dose of CI to the date of progression or censoring of data 
$\uparrow$ Overall survival was calculated from the date of first dose of CI to the date of death or censoring of data

Extended Data Table 2. Patient characteristics of the Leuven cohort

\begin{tabular}{ccccc} 
Patient number & Orig.ident & Tissue & Library type & Stage \\
\hline P1 & sc5rCMA070 & Subcutis & 5' GEX $^{\prime}$ & IV \\
P2 & scrCMA036 & Subcutis & 3' GEX & IV \\
P3 & scrCMA038 & Lymph node & 3' GEX & IV \\
P4.1 & scrCMA040 & Skin & 3' GEX & IV \\
P4.2 & scrCMA041 & Skin & 3' GEX & IV \\
P5 & scrCMA054 & Lymph node & 3' GEX & IV \\
P6 & scrCMA055 & Lymph node & 3' GEX & IIIC \\
P7 & scrCMA068 & Subcutis & 3' GEX & IV \\
P8 & scrCMA076 & Lymph node & 3' GEX & IV \\
P9 & scrCMA087 & Lymph node & 3' GEX & IV \\
P10 & scrCMA090 & Subcutis & 3' GEX & IV \\
P11 & scrCMA093 & Lymph node & 3' GEX & IIIC \\
P12 & scrCMA094 & Lymph node & 3' GEX & IV \\
P13 & scrCMA109 & Skin & 3' GEX & IIIC \\
P14 & scrCMA119 & Lymph node & 3' GEX & IIID \\
P15 & scrCMA120 & Skin & 3' GEX & IV \\
\hline
\end{tabular}


Extended Data Table 3. Patient characteristics of the Zurich (ZH) Cohort

Characteristic

$$
\begin{array}{cc}
\text { Disease-Free } & \text { Relapse } \\
(\mathrm{N}=19, \mathbf{6 3 \%}) & (\mathrm{N}=\mathbf{1 1}, \mathbf{3 7 \%})
\end{array}
$$

\begin{tabular}{lcc}
\hline Age at start of treatment - years & 62 & 67 \\
$\quad$ Median & $22-78$ & $35-87$ \\
$\quad$ Range & & \\
\hline Sex - no. of patients (\%) & $9(47.4)$ & $7(63.6)$ \\
$\quad$ Female & $10(52.6)$ & $4(36.4)$ \\
$\quad$ Male & $14(73.7)$ & $3(27.3)$ \\
\hline BRAF mutation - no. of patients (\%) & $5(26.3)$ & $8(72.7)$ \\
$\quad$ No & 615 & 77 \\
$\quad$ Yes & $191-835$ & $31-373$ \\
\hline Progression-free survival - days* & & \\
$\quad$ Median & 615 & 540 \\
$\quad$ Range & $191-856$ & $170-895$ \\
\hline Overall survival - days ${ }^{\dagger}$ & & \\
$\quad$ Median &
\end{tabular}

779

$780 *$ Progression-free survival was calculated from the date of first dose of CI to the date of progression or 781 censoring of data

782

$\dagger$ Overall survival was calculated from the date of first dose of CI to the date of death or censoring of data 
784 Extended Data Table 4. Gene signatures based on Tsoi et al. ${ }^{33}$

\begin{tabular}{|c|c|c|c|}
\hline Undifferentiated & Neural crest- like & Transitory & Melanocytic \\
\hline$A J U B A$ & $V I T$ & $X Y L T 1$ & $C C D C 171$ \\
\hline TOR4A & $V I P R I$ & TSPAN7 & CFAP61 \\
\hline MARCH4 & $V E G F C$ & SOD3 & ZDHНC11B \\
\hline ZDHHC2 & TWIST2 & $S C R G 1$ & VEPHI \\
\hline ZNF467 & TNFRSF12A & SORL1 & TNFRSF14 \\
\hline ZNF185 & $T P M I$ & SEMA3E & $T D R D 3$ \\
\hline ZIC2 & $T P B G$ & SELENBPI & $T P P P$ \\
\hline$V A S N$ & TLE4 & RNASE1 & TRIM63 \\
\hline$U C P 2$ & TOX2 & RAPGEF4 & TRPM1 \\
\hline GALNT6 & TLR4 & PCDH7 & ТTC39A \\
\hline TNFAIP2 & THSD4 & PRSS33 & TSPAN1O \\
\hline TNFSF 18 & STX1A & PCSK6 & SLC7A8 \\
\hline TMEM4O & SYT1 & PLBD1 & SLC16A6 \\
\hline TMEM200A & SYNPO & NELL1 & SLAMF7 \\
\hline TMEM184A & STRA6 & $N P R I$ & SEMA6A \\
\hline$T B L 1 X$ & STC2 & MCAM & $R U N X 3$ \\
\hline TRERF1 & SPRED3 & MMP15 & $R N F 144 B$ \\
\hline TOX & SPOCD1 & $M A M D C 2$ & $R N L S$ \\
\hline$T B C 1 D 2$ & SPOCK1 & LSAMP & $R G S 12$ \\
\hline$S F N$ & $S L C 2 A 1$ & LRRTM4 & PYCARD \\
\hline SAMD12 & SLC16A2 & GDF11 & PRUNE2 \\
\hline SAMD11 & SLC14A1 & FXYD3 & $P R K C B$ \\
\hline SOX9 & $S L C 12 A 8$ & $E B F 3$ & PRDM7 \\
\hline SLC8A1 & $S M A G P$ & COL11A2 & KCNAB2 \\
\hline SLC $38 A 4$ & SLIT2 & COL9A1 & $O C A 2$ \\
\hline SLC16A14 & $S D K 1$ & CX3CL1 & $N R 4 A 3$ \\
\hline SCN5A & STAC & BCHE & $N A V 2$ \\
\hline SCNNIA & SLFN11 & ANO4 & MYOID \\
\hline SH3RF2 & $S 100 A 2$ & $A L D H 1 A 1$ & MAPK4 \\
\hline SERPINB7 & $\mathrm{ROBO} 4$ & & MATIA \\
\hline SLPI & $R A B 27 B$ & & MLANA \\
\hline SECTMI & PKIA & & $L X N$ \\
\hline$R U N X 2$ & PRSS23 & & $K C P$ \\
\hline ARHGAP29 & $P A P P A$ & & IL16 \\
\hline REN & PRDMI & & $I L 12 R B 2$ \\
\hline$P A W R$ & KCNMAl & & $H S D 17 B 14$ \\
\hline PSG9 & KCNN4 & & HMOX1 \\
\hline PSG5 & $P O D X L$ & & $H 2 A F J$ \\
\hline PSG4 & $P D G F R B$ & & $G O L G A 7 B$ \\
\hline$P B X 1$ & PLAUR & & $Q P C T$ \\
\hline PLAGLI & $P X D N$ & & GFOD1 \\
\hline PHLDB2 & РTХ3 & & GPRI43 \\
\hline
\end{tabular}




\begin{tabular}{|c|c|c|}
\hline PLEKHA6 & NMNAT2 & $F Y B$ \\
\hline$P D G F C$ & $N R P 1$ & FAM $83 \mathrm{H}$ \\
\hline$P L A U$ & $N G E F$ & $F A M 174 B$ \\
\hline$P K P 2$ & $N E G R 1$ & EPHA5 \\
\hline PLAC8 & $N R G 1$ & ENTHDI \\
\hline$P A D I 3$ & NTN4 & DNAJA4 \\
\hline PITX1 & $M T 2 A$ & $D E N N D 2 D$ \\
\hline$N U A K 1$ & MTIE & C2orf88 \\
\hline$N T N G 1$ & MPP4 & CCL18 \\
\hline NMT2 & $L O X L 2$ & CEACAMI \\
\hline MYEOV & LDOCl & $C A P G$ \\
\hline MICAL2 & $L A M B 3$ & $\mathrm{CDH3}$ \\
\hline MGST1 & $J U N$ & $\mathrm{CDHl}$ \\
\hline МЕСOM & IL31RA & ATP6VOD2 \\
\hline LYPD6B & $I L 11$ & $A B C D 1$ \\
\hline LAMA5 & $I L I B$ & $A B C B 5$ \\
\hline KISSI & ITGA3 & $A P O L D 1$ \\
\hline KRT86 & $I T G A 2$ & $A N K R D 30 B$ \\
\hline KRT81 & IGFBP6 & $A D C Y 2$ \\
\hline KRT80 & $I D 1$ & $A D A M 23)$ \\
\hline KRT8 & $I N H B A$ & \\
\hline KRT7 & $H R H I$ & \\
\hline KRT18 & GAS6 & \\
\hline$J U P$ & GLIPRI & \\
\hline$I L 7 R$ & GFRAl & \\
\hline$I L 4 R$ & GATA3 & \\
\hline IRSI & GPR176 & \\
\hline IGFN1 & $F Z D 2$ & \\
\hline HES7 & FJXI & \\
\hline$G D A$ & FOSL1 & \\
\hline GLIS2 & FOXF1 & \\
\hline GATA2 & FBLIMI & \\
\hline$G P R C 5 C$ & $F L N B$ & \\
\hline GPRC5A & $F A M 83 G$ & \\
\hline$F M N L 1$ & FAM20C & \\
\hline FOXAl & FAM171A1 & \\
\hline$F L N C$ & FAM155A & \\
\hline FERMTI & ERRFII & \\
\hline FAT4 & EFNB2 & \\
\hline FAM196B & $D P Y D$ & \\
\hline ELFN2 & $D K K 1$ & \\
\hline$E G F R$ & DOCK5 & \\
\hline$D S E$ & CYR61 & \\
\hline$D M B T 1$ & $C L M P$ & \\
\hline
\end{tabular}




\begin{tabular}{|c|c|}
\hline DIO2 & COL13A1 \\
\hline DOCK2 & COL12A1 \\
\hline CYP2S1 & COL5Al \\
\hline CRIMI & $F 2 R L 2$ \\
\hline CDK15 & C16orf45 \\
\hline CORO6 & C15orf52 \\
\hline COLECIO & C12orf75 \\
\hline CCDC $88 C$ & $C D 163 L 1$ \\
\hline CCDC69 & CAVI \\
\hline$F 3$ & CARD10 \\
\hline$F 2 R L 1$ & $C L C F 1$ \\
\hline$C L U$ & $\mathrm{CDH} 13$ \\
\hline CDYL2 & $B M P 2$ \\
\hline CITED2 & $A X L$ \\
\hline CARDI1 & $A B C C 3$ \\
\hline CPA4 & $A R N T L 2$ \\
\hline CREB3L1 & ANTXR2 \\
\hline CNN1 & ANXAl \\
\hline$C A L B 2$ & $A K R 1 C 3$ \\
\hline $\mathrm{CDH} 4$ & $A R L A C$ \\
\hline \multicolumn{2}{|l|}{ BTBD11 } \\
\hline \multicolumn{2}{|l|}{$B D N F$} \\
\hline \multicolumn{2}{|l|}{$B A S P 1$} \\
\hline \multicolumn{2}{|l|}{$B N C 1$} \\
\hline \multicolumn{2}{|l|}{ ATP $8 B 1$} \\
\hline \multicolumn{2}{|l|}{$A B C G 2$} \\
\hline \multicolumn{2}{|l|}{$A R M C 4$} \\
\hline \multicolumn{2}{|l|}{$A N K R D I$} \\
\hline \multicolumn{2}{|l|}{$A R$} \\
\hline \multicolumn{2}{|l|}{$A M I G O 2$} \\
\hline ADAMTSL1 & \\
\hline$A C S L 5$ & \\
\hline
\end{tabular}

785

786 
787 Extended Data Table 5. Gene signatures based on Rambow et al. ${ }^{34}$

\begin{tabular}{|c|c|c|}
\hline Pigmentation & HALLMARK_EMT & NSCS \\
\hline$T Y R$ & $A B I 3 B P$ & $I G F 1$ \\
\hline$S L C 45 A 2$ & АCTA2 & $C A D M 1$ \\
\hline$P M E L$ & $A D A M 12$ & $V C A N$ \\
\hline SLC24A5 & ANPEP & ADAMTS4 \\
\hline$S 100 B$ & $A P L P 1$ & $P D G F B$ \\
\hline$M L A N A$ & $A R E G$ & ITGA6 \\
\hline GPR143 & $B A S P 1$ & SYT11 \\
\hline$M L P H$ & $B D N F$ & COLAAI \\
\hline$R A B 38$ & $B G N$ & $A Q P 1$ \\
\hline MITF & $B M P 1$ & $A T P 1 B 2$ \\
\hline$T Y R P 1$ & $C A D M 1$ & $A D G B$ \\
\hline$D C T$ & $C A L D 1$ & TMEM176B \\
\hline$R A B 27 A$ & $C A L U$ & $S L C 22 A 17$ \\
\hline$E D N R B$ & $C A P 2$ & $N R X N 1$ \\
\hline$K I T$ & $C A P G$ & MATN2 \\
\hline \multirow[t]{27}{*}{$T R P M 1$} & $C D 44$ & GFRAl \\
\hline & CD59 & $M P Z$ \\
\hline & $\mathrm{CDH11}$ & $N G F R$ \\
\hline & $\mathrm{CDH} 2$ & PRIMAI \\
\hline & CDH6 & $L A M C C 1$ \\
\hline & COL11A1 & RSPO3 \\
\hline & COL12A1 & ITGAI \\
\hline & COL16A1 & THBS2 \\
\hline & COLlA1 & $N L G N 3$ \\
\hline & COLIA2 & GFRA3 \\
\hline & COL3A1 & S100A4 \\
\hline & COLAAl & $C N N 3$ \\
\hline & $C O L 4 A 2$ & $A N X A 1$ \\
\hline & COL5A1 & SLITRK6 \\
\hline & COL5A2 & $A 2 M$ \\
\hline & COL5A3 & COLIAl \\
\hline & COL6A2 & LICAM \\
\hline & COL6A3 & SEMA3B \\
\hline & COL7A1 & GFRA2 \\
\hline & COL8A2 & ATP1A2 \\
\hline & COMP & $I L I R A P$ \\
\hline & COPA & PLAT \\
\hline & $C R L F 1$ & SOX10 \\
\hline & $C T G F$ & $G D N F$ \\
\hline & CTHRC1 & $R X R G$ \\
\hline & $C X C L 1$ & $M E F 2 C$ \\
\hline & CXCL12 & $T F A P 2 B$ \\
\hline
\end{tabular}




CXCL6
CYR61
DAB2
DCN
DKK1
DPYSL3
DST
ECM1
ECM2
EDIL3
EFEMP2
ELN
EMP3
ENO2
FAP
FAS
FBLN1
FBLN2
FBLN5
FBN1
FBN2
FERMT2
FGF2
FLNA
FMOD
FN1
FOXC2
FSTL1
FSTL3
FUCA1
FZD8
GADD45A
GADD45B
GAS1
GEM
GJA1
GLIPR1
GLT25D1
GPC1
GPX7
GREM1
ITRA1

IGFBP 2 


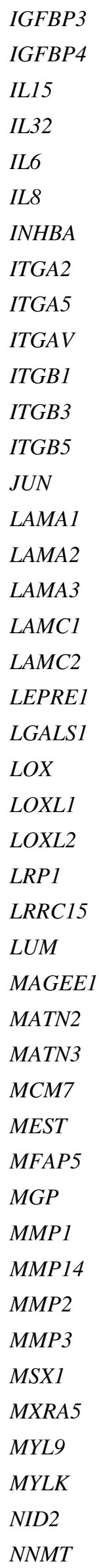

NNMT 


NOTCH2
NT5E
NTM
OXTR
PCOLCE
PCOLCE2
PDGFRB
PDLIM4
PFN2
PLAUR
PLOD1
PLOD2
PLOD3
PMEPA1
PMP22
POSTN
PPIB
PRRX1
PRSS2
PTHLH
PTX3
PVR
QSOX1
RGS4
RHOB
SAT1
SCG2
SDC1
SDC4
SERPINE1
SERPINE2
SERPINH1
SFRP1
SFRP4
SGCB
SGCD
SGCG
SLC6A8
SLIT2
SLIT3
SNAI2
SNTB1
SPARC
SPOCK1




SPP1
TAGLN
TFPI2
TGFB1
TGFBI
TGFBR3
TGM2
THBS1
THBS2
THY1
TIMP1
TIMP3
TNC
TNFAIP3
TNFRSF11B
TNFRSF12A
TPM1
TPM2
TPM4
VCAM1
VCAN
VEGFA
VEGFC
VIM
WIPF1
WNT5A

788 
a

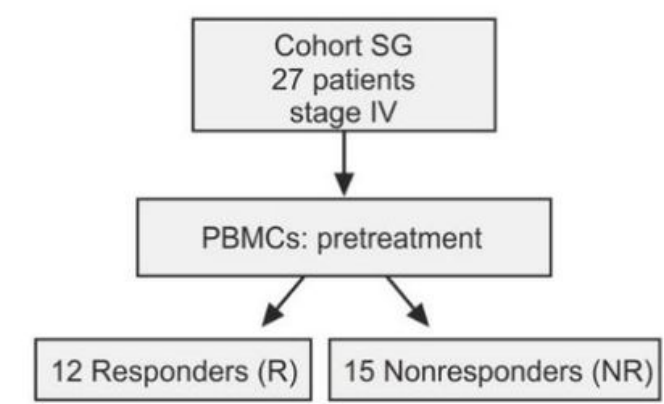

b

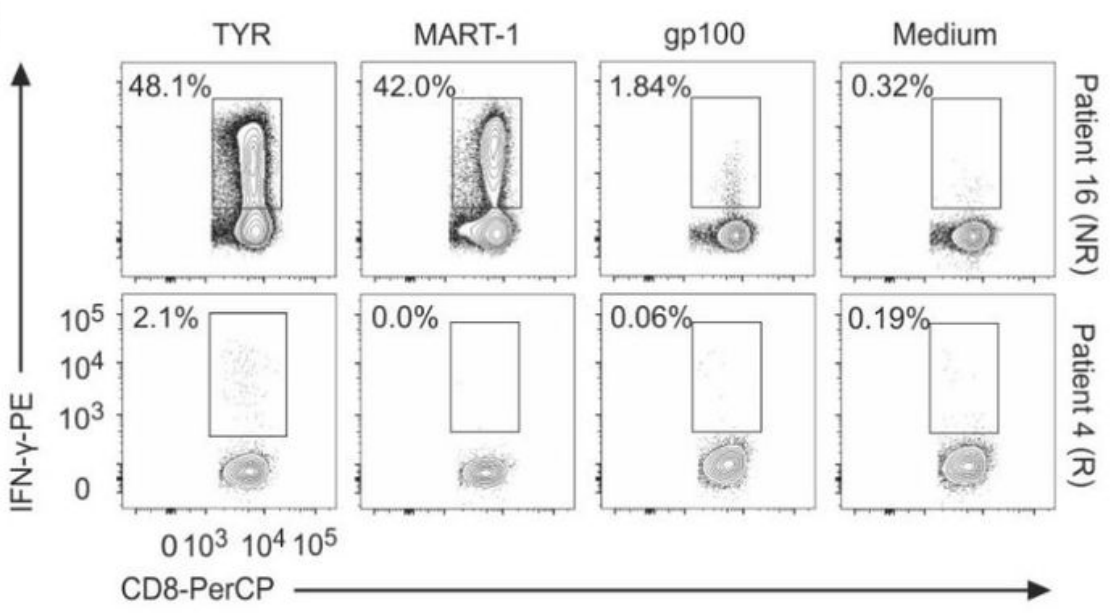

C

Patient ID

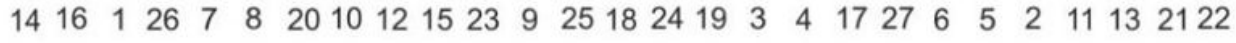
IFN- $\mathrm{\gamma}^{+} \mathrm{CD}^{+} \mathrm{T}$ cells TYR MART-1 gp100

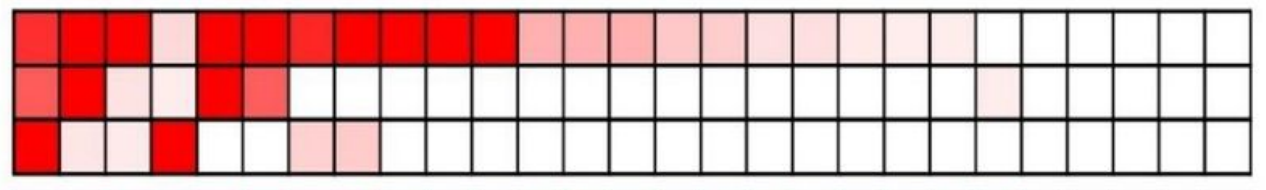

Breadth of $\mathrm{T}$ cell response Response to $\mathrm{Cl}$ therapy
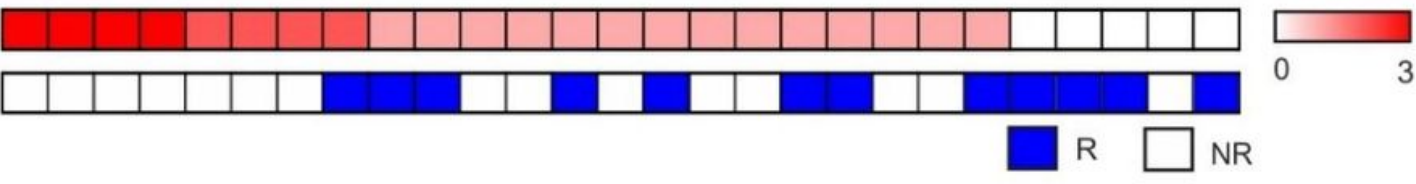

d

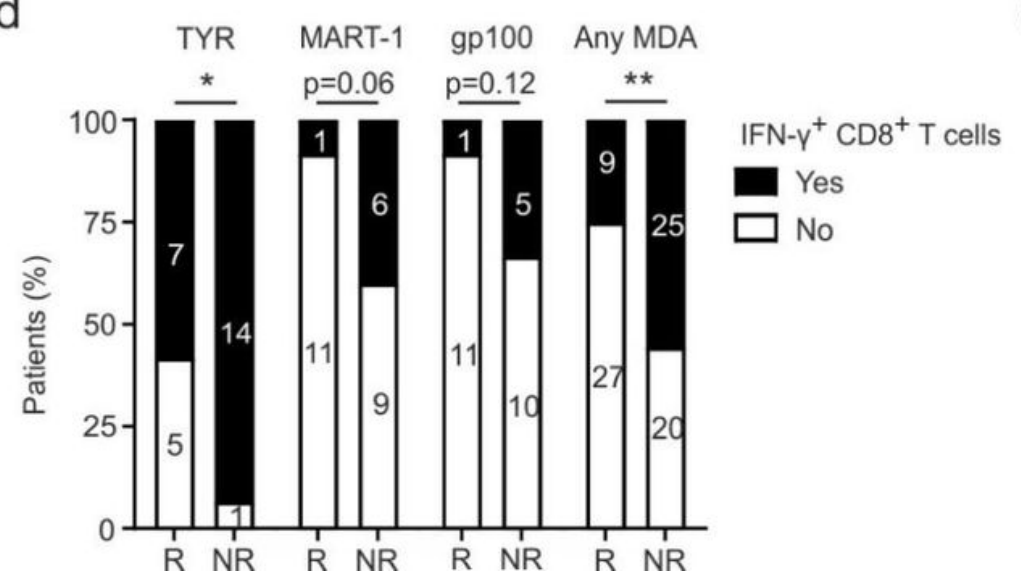

e

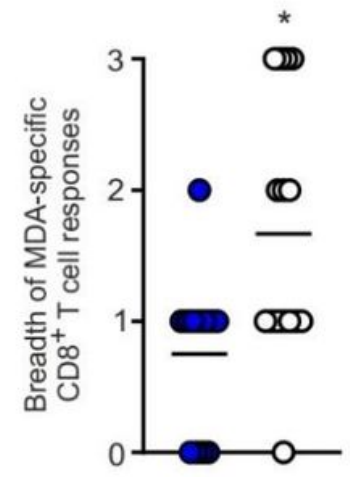

f

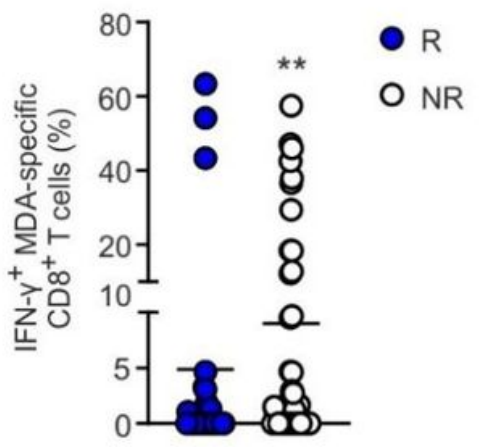

Figure 1

Levels of pre-existing MDA-specific CD8+ T cells in melanoma patients receiving checkpoint inhibitor therapy. a, In the prospective St.Gallen (SG) cohort, peripheral blood mononuclear 2cells (PBMCs) were obtained from 27 patients with stage IV melanoma prior to checkpoint inhibitor therapy. Clinical responders $(R ; N=12)$ and nonresponders $(N R ; N=15)$ were determined at 12 weeks. b-f, CD8+ T cell responses against the melanocyte differentiation antigens (MDAs) TYR, MART-1 and gp100 after in vitro stimulations of PBMCs with overlapping 15-mer peptide pools for each individual antigen for 10 days as measured by IFN-y expression. b, Representative FACS plots of samples from patient 16 (NR) and patient 
4 (R). c, Heatmaps summarizing the frequency of IFN- $y$-producing CD8+ T cells for the indicated antigens. The color scale represents the frequency of IFN- $\gamma+\mathrm{CD} 3+\mathrm{CD} 8+\mathrm{CD} 45 \mathrm{RAlow} T$ cells. The breadth of the CD8 $+T$ cell response was defined as the number of the individual IFN- $\gamma+C D 8+T$ cells against the MDAs TYR, MART-1 and gp100, as indicated by the detection of IFN- -+ CD8+ T cell responses, ranging from 0 (none) to 3 (all). $d$, Proportions of $R$ and NR with MDA-specific CD8+ $T$ cell responses to the individual MDAs or any MDA. e, Summary of the breadth of MDA-specific CD8+ T cells in R and NR as shown for individual patients as shown in panel c. f, Frequencies of IFN- $y+C D 8+T$ cells against any MDA (TYR, MART-1 or gp100) in R vs NR. Dots represent individual patients, and lines indicate the mean values (e and f). Statistical analysis was performed using the chi-square test (d) and Mann-Whitney test (e and f); ${ }^{*} \mathrm{P}<0.05, * * \mathrm{P}<0.01, * * * \mathrm{P}<0.001$.

a

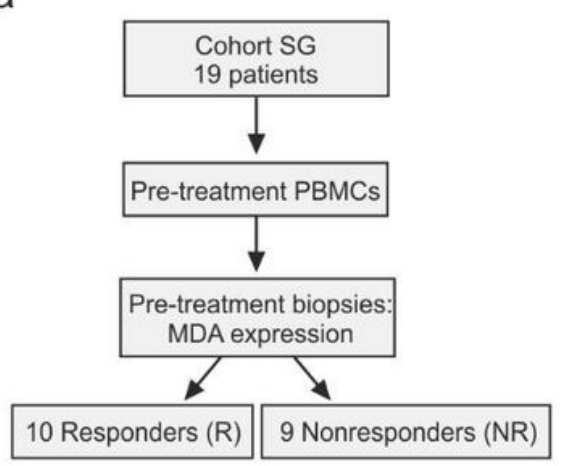

b

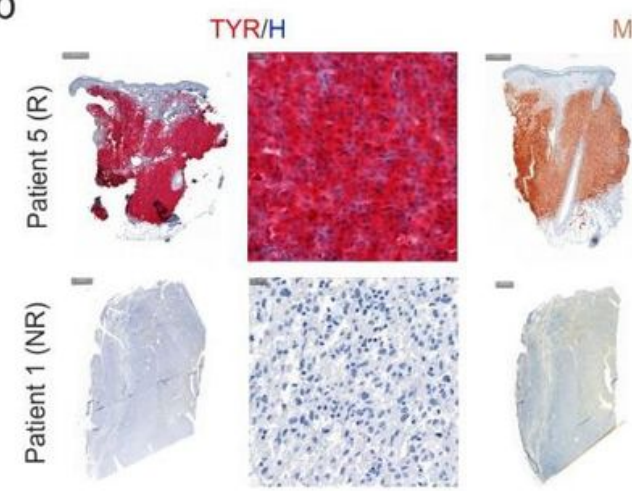

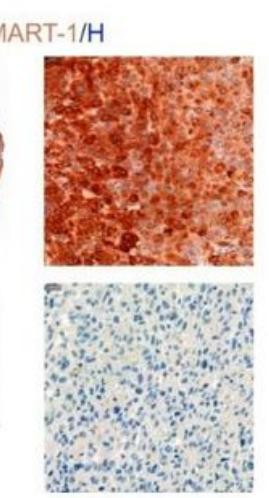

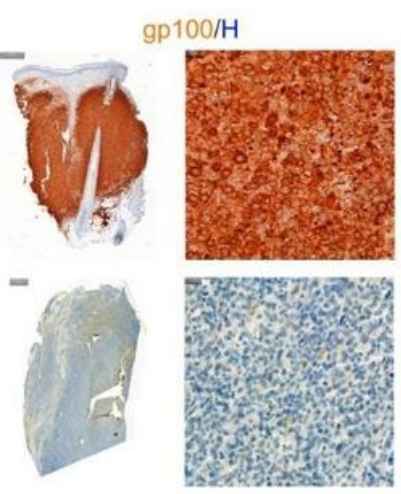

C

Patient ID $\begin{array}{lllllllllllllllllll}14 & 1 & 8 & 20 & 10 & 12 & 15 & 23 & 25 & 18 & 19 & 3 & 4 & 17 & 5 & 2 & 11 & 21 & 22\end{array}$

Breadth of T cell response

TYR

MART-1

gp100

Breadth of MDA expression

Response to $\mathrm{Cl}$ therapy
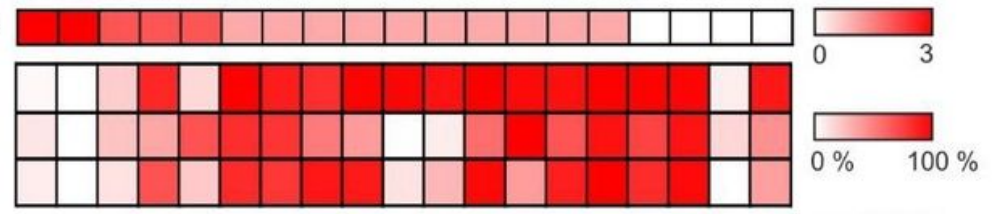

d

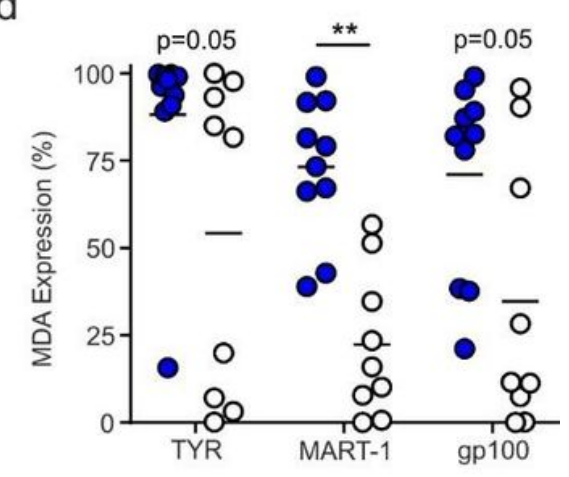

e

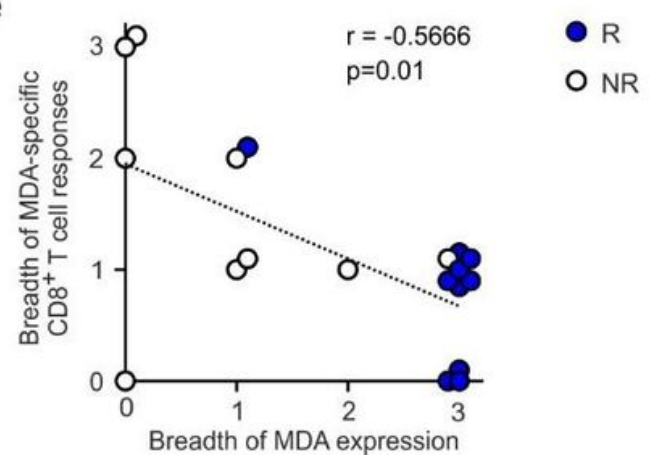

Figure 2

MDA tissue expression inversely correlates with peripheral MDA-specific CD8+ T cells. a, In 19 of the patient from the SG cohort, tumor biopsies obtained before initiation of checkpoint inhibitor therapy and 
matched PBMCs from Fig. 1 were analyzed. The expression of the MDAs TYR, MART-1 and gp100 was analyzed using quantitative IHC analysis of biopsy tissue. b, Representative images for the indicated antigens in patient $5(\mathrm{R})$ and patient $1(\mathrm{NR})$. The frequency of tumor cells expressing each of these antigens was assessed as outlined in Extended Data Figure 3. Hematoxylin $(H)$ staining to identify the cell nucleus. Scale bars are 500 and $2000 \mu \mathrm{m}$ for the general images (top and bottom images) and $20 \mu \mathrm{m}$ for the magnified images. $c$, Heatmaps summarizing the frequency of melanoma cells stained positive for the MDAs TYR, MART-1 and gp100. The breadth of MDA expression was defined as the number of MDAs expressed per patient sample ranging from 0 (none) to 3 (all) as defined by values from ROC curves for each individual antigen. The breadth of T cell response (data from Fig. 1) is specified as the number of individual MDAs recognized, as indicated by the detection of IFN- $\gamma+\mathrm{CD} 8+\mathrm{T}$ cell responses, ranging from 0 (none) to 3 (all). d, Frequencies of melanoma cells showing positive staining for each of the MDAs TYR, MART-1 and gp100 in the analyzed tissue slides between R and NR. Dots represent individual patients, and lines indicate mean values. e, Correlation between the breadth of MDA expression and that of MDA-specific CD8+ T cell responses. Dots represent individual patients. Statistical analysis was performed using the Mann-Whitney test (d) and Spearman's correlation (e); ${ }^{P}<0.05,{ }^{\star \star} P<0.01$, ${ }^{\star \star \star} P$ $<0.001$. 

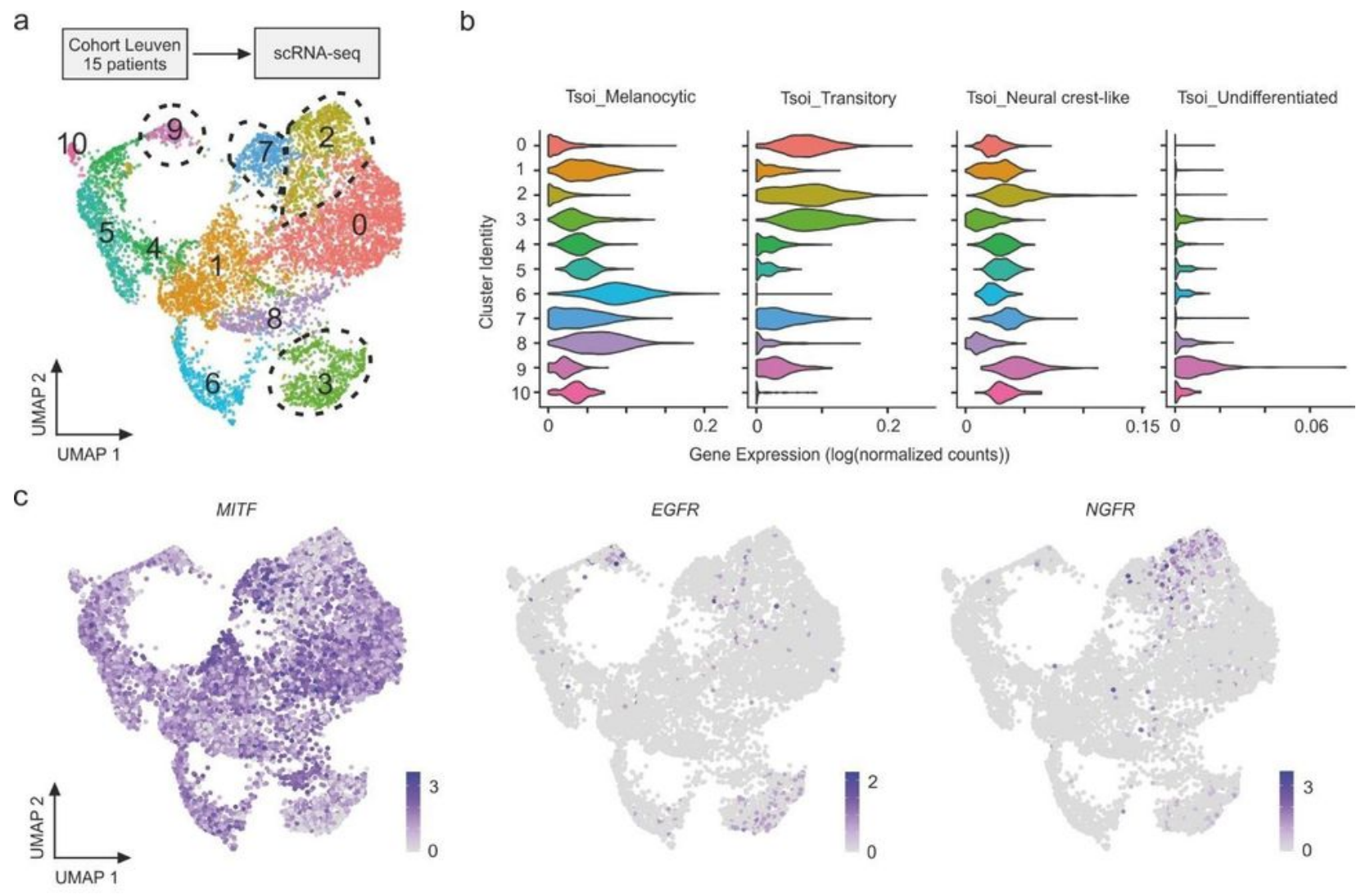

d

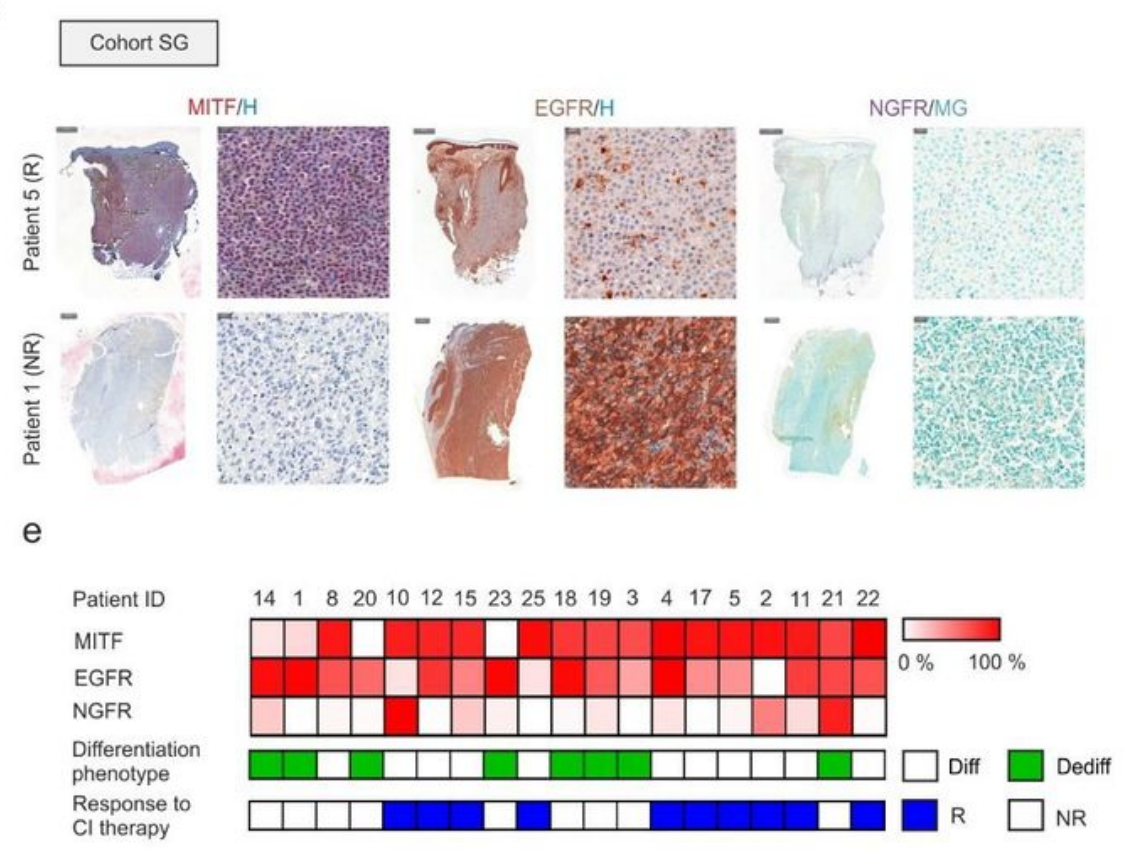

f
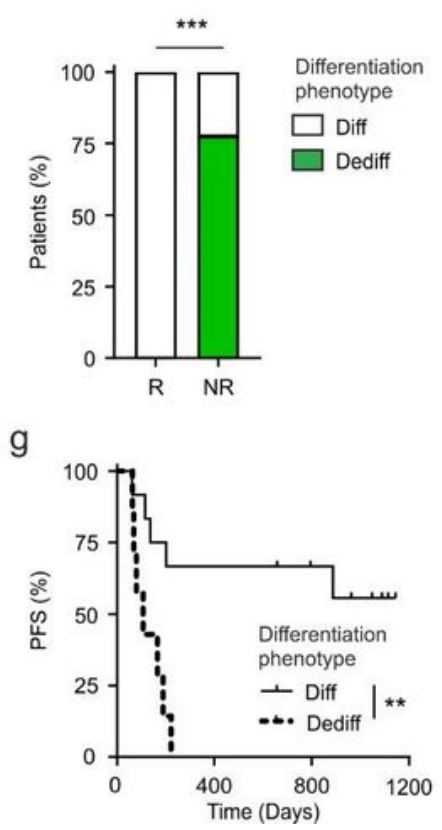

\section{Figure 3}

The dedifferentiated melanoma phenotype predicts patient outcome in response to checkpoint inhibitor therapy. a-c, We collected melanoma metastases from 15 stage III or stage IV treatment-naïve patients and performed single-cell RNA-seq analysis of tumor cells (Leuven cohort). a, UMAP plot displaying cluster assignment. Highlighted clusters harbor tumor cells with a dedifferentiated phenotype (cluster 2, 3,7 and 9) whereas the other cluster exhibit a melanocytic and differentiated phenotype. b, Violin plots 
based on the Tsoi et el. (2018) gene signatures for melanocytic, transitory, neural crest-like and undifferentiated melanoma phenotypes. c, UMAP plots showing the gene expression of MITF, EGFR and NGFR. d-g, In the SG cohort, the expression of MITF, EGFR and NGFR was assessed 290 in tumor biopsies obtained before initiation of checkpoint inhibitor therapy by quantitative IHC analysis. $d$, Representative images for the indicated antigens in patient $5(R)$ and patient $1(N R)$. The frequency oftumor cells expressing each of these antigens was assessed as outlined in Extended Data Figure 3. Hematoxylin $(\mathrm{H})$ or Methyl Green (MG) staining to identify the cell nucleus. Scale bars are 500 and $2000 \mu \mathrm{m}$ for the general images (top and bottom images) and $20 \mu \mathrm{m}$ for the magnified images. e, Heatmaps summarizing the frequencies of melanoma cells showing positive staining for MITF, EGFR or NGFR using quantitative IHC analysis. The dedifferentiated melanoma phenotype (Dediff) was defined as MITFlow EGFRhigh or MITFlow NGFRhigh as based on threshold values from ROC curves for each individual protein. $f$, Proportions of patients in the 19 patients from the SG cohort with a dedifferentiated phenotype in the R and NR groups. g, Progression-free survival of patients with a dedifferentiated or differentiated melanoma phenotype. Statistical analysis was performed using the chi-square test $(f)$ or the Mantel-Cox log-rank test $(\mathrm{g}) ;{ }^{*} \mathrm{P}<0.05, * * \mathrm{P}<0.01,{ }^{* * *} \mathrm{P}<0.001$.

a

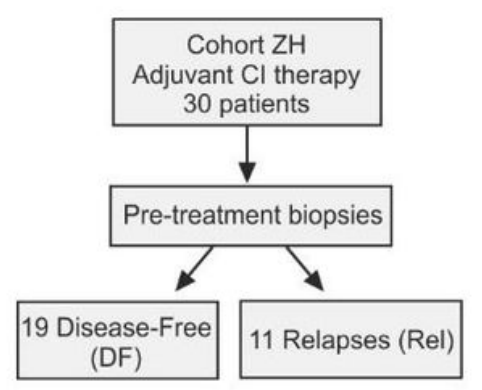

$\mathrm{b}$

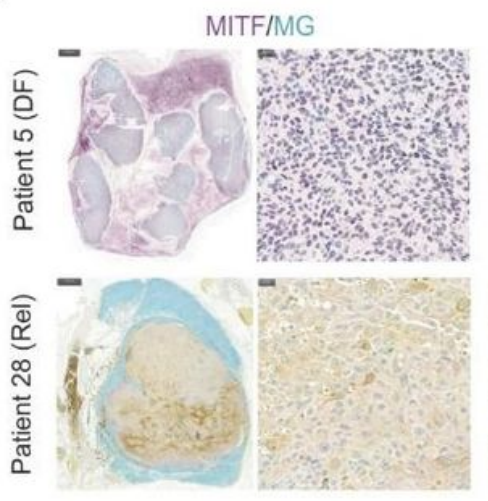

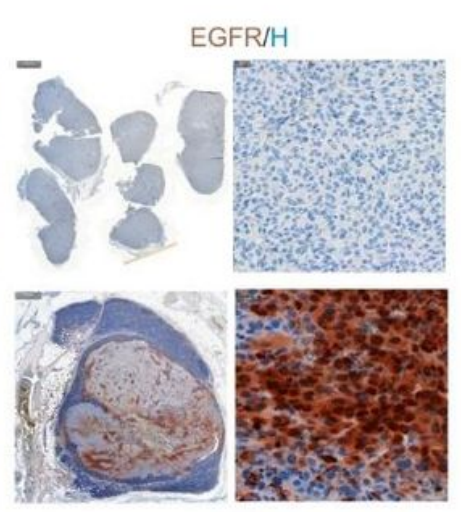

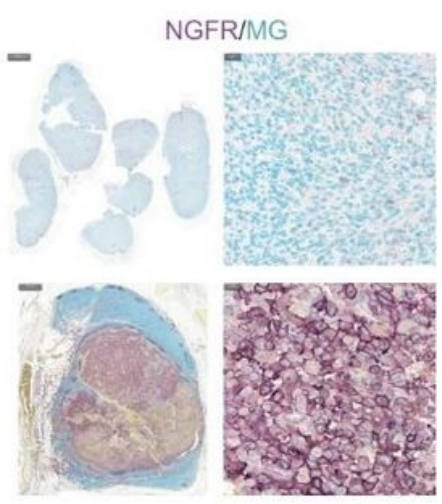

C

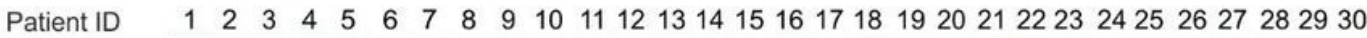
MITF

EGFR NGFR

Differentiation phenotype

Response to $\mathrm{Cl}$ therapy
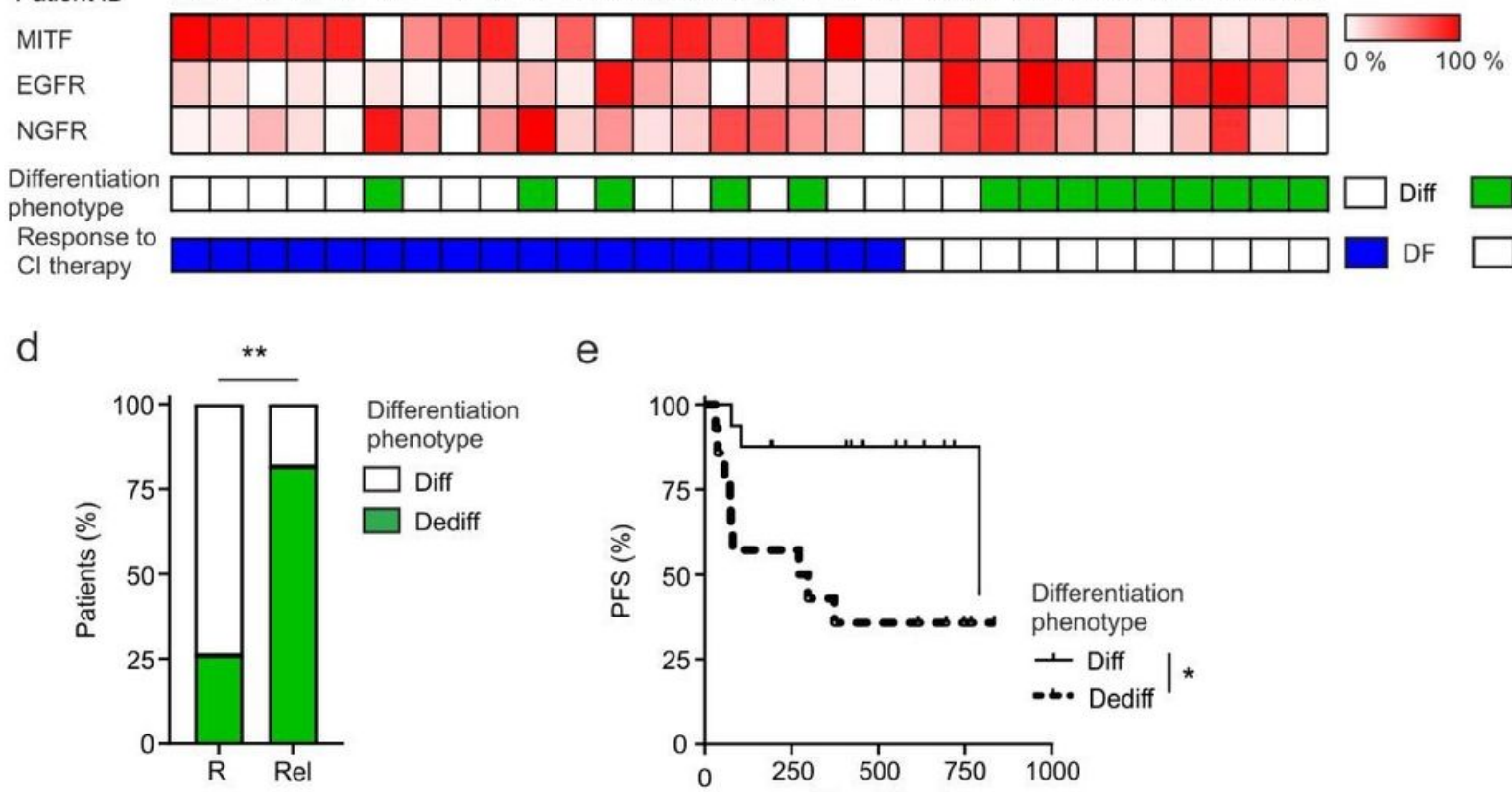

e

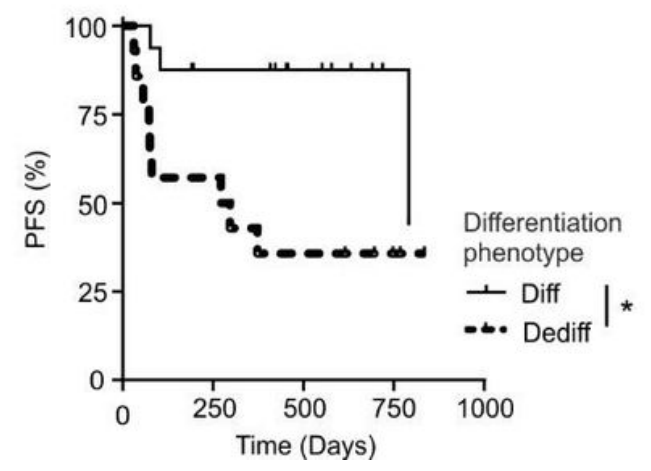




\section{Figure 4}

The dedifferentiated melanoma phenotype predicts patient outcome in response to adjuvant anti-PD-1 therapy. a, In the Zurich (ZH) adjuvant cohort, tumor biopsies were obtained before initiation of adjuvant anti-PD-1 treatment. Disease-Free patients (DF; $R ; n=19)$ and patients who experienced relapse (Rel; $n=11)$ were determined at the first PET-CT scan within 3 months after start of therapy. b, Representative images showing the expression of MITF, EGFR and NGFR in patient 5 (DF) and patient 28 (Rel). The frequency of tumor cells expressing each of these antigens was assessed as outlined in Extended Data Figure 3. Hematoxylin (H) or Methyl Green (MG) staining to identify the cell nucleus. Scale bars are 1000 and 2000 $\mu \mathrm{m}$ for the general images (top and bottom images) and $20 \mu \mathrm{m}$ for the magnified images. c, Heatmaps summarizing the frequency of tumor cells stained positive for MITF, EGFR and NGFR using quantitative IHC analysis. The dedifferentiated phenotype (Dediff) was defined as MITFlow EGFRhigh or MITFlow NGFRhigh and based on threshold values from ROC curves for each individual protein. d, Proportions of patients in the ZH adjuvant anti-PD-1 cohort with a dedifferentiated phenotype in the DF 318 and Rel groups. e, Progression-free survival of patients with a dedifferentiated or differentiated melanoma phenotype. Statistical analysis was performed using the chi-square test (d) or Mantel-Cox log-rank test (e); $* \mathrm{P}<0.05,{ }^{*} \mathrm{P}<0.01, \star \star \star \mathrm{P}<0.001$. 